\title{
The Boundedness of Intrinsic Square Functions on the Weighted Herz Spaces
}

\author{
Hua Wang \\ College of Mathematics and Econometrics, Hunan University, Changsha 410082, China \\ Correspondence should be addressed to Hua Wang; wanghua@pku.edu.cn \\ Received 23 September 2013; Accepted 4 November 2013; Published 12 January 2014 \\ Academic Editor: Dachun Yang \\ Copyright (c) 2014 Hua Wang. This is an open access article distributed under the Creative Commons Attribution License, which \\ permits unrestricted use, distribution, and reproduction in any medium, provided the original work is properly cited. \\ We will obtain the strong type and weak type estimates of intrinsic square functions including the Lusin area integral, Littlewood- \\ Paley $\mathscr{G}$-function, and $\mathscr{G}_{\lambda}^{*}$-function on the weighted Herz spaces $\dot{K}_{q}^{\alpha, p}\left(w_{1}, w_{2}\right)\left(K_{q}^{\alpha, p}\left(w_{1}, w_{2}\right)\right)$ with general weights.
}

\section{Introduction and Main Results}

Let $\mathbb{R}_{+}^{n+1}=\mathbb{R}^{n} \times(0, \infty)$ and $\varphi_{t}(x)=t^{-n} \varphi(x / t)$. The classical square function (Lusin area integral) is a familiar object. If $u(x, t)=P_{t} * f(x)$ is the Poisson integral of $f$, where $P_{t}(x)=\left(c_{n}\right)\left(t /\left(t^{2}+|x|^{2}\right)^{(n+1) / 2}\right)$ denotes the Poisson kernel in $\mathbb{R}_{+}^{n+1}$, then we define the classical square function (Lusin area integral) $S(f)$ by (see $[1,2])$

$$
S(f)(x)=\left(\iint_{\Gamma(x)}|\nabla u(y, t)|^{2} t^{1-n} d y d t\right)^{1 / 2},
$$

where $\Gamma(x)$ denotes the usual cone of aperture one:

$$
\begin{gathered}
\Gamma(x)=\left\{(y, t) \in \mathbb{R}_{+}^{n+1}:|x-y|<t\right\}, \\
|\nabla u(y, t)|^{2}=\left|\frac{\partial u}{\partial t}\right|^{2}+\sum_{j=1}^{n}\left|\frac{\partial u}{\partial y_{j}}\right|^{2} .
\end{gathered}
$$

Similarly, we can define a cone of aperture $\gamma$ for any $\gamma>0$ :

$$
\Gamma_{\gamma}(x)=\left\{(y, t) \in \mathbb{R}_{+}^{n+1}:|x-y|<\gamma t\right\},
$$

and corresponding square function

$$
S_{\gamma}(f)(x)=\left(\iint_{\Gamma_{\gamma}(x)}|\nabla u(y, t)|^{2} t^{1-n} d y d t\right)^{1 / 2} .
$$

The Littlewood-Paley $g$-function (could be viewed as a "zeroaperture" version of $S(f)$ ) and the $g_{\lambda}^{*}$-function (could be viewed as an "infinite aperture" version of $S(f))$ are defined, respectively, by (see, e.g., $[3,4]$ )

$$
\begin{gathered}
g(f)(x)=\left(\int_{0}^{\infty}|\nabla u(x, t)|^{2} t d t\right)^{1 / 2}, \\
g_{\lambda}^{*}(f)(x)=\left(\iint_{\mathbb{R}_{+}^{n+1}}\left(\frac{t}{t+|x-y|}\right)^{\lambda n}\right. \\
\left.\times|\nabla u(y, t)|^{2} t^{1-n} d y d t\right)^{1 / 2},
\end{gathered}
$$

$\lambda>1$.

The modern (real-variable) variant of $S_{\gamma}(f)$ can be defined in the following way (here we drop the subscript $\gamma$ if $\gamma=1)$. Let $\psi \in C^{\infty}\left(\mathbb{R}^{n}\right)$ be real, radial and have support contained in $\{x:|x| \leq 1\}$, and let $\int_{\mathbb{R}^{n}} \psi(x) d x=0$. The continuous square function $S_{\psi, \gamma}(f)$ is defined by (see, e.g., $[5,6])$

$$
S_{\psi, \gamma}(f)(x)=\left(\iint_{\Gamma_{\gamma}(x)}\left|f * \psi_{t}(y)\right|^{2} \frac{d y d t}{t^{n+1}}\right)^{1 / 2} .
$$

In 2007, Wilson [7] introduced a new square function called intrinsic square function which is universal in a sense (see also [8]). This function is independent of any particular kernel $\psi$, and it dominates pointwise all the above-defined 
square functions. On the other hand, it is not essentially larger than any particular $S_{\psi, \gamma}(f)$. For $0<\beta \leq 1$, let $\mathscr{C}_{\beta}$ be the family of functions $\varphi$ defined on $\mathbb{R}^{n}$ such that $\varphi$ has support containing in $\left\{x \in \mathbb{R}^{n}:|x| \leq 1\right\}, \int_{\mathbb{R}^{n}} \varphi(x) d x=0$, and for all $x, x^{\prime} \in \mathbb{R}^{n}$,

$$
\left|\varphi(x)-\varphi\left(x^{\prime}\right)\right| \leq\left|x-x^{\prime}\right|^{\beta}
$$

For $(y, t) \in \mathbb{R}_{+}^{n+1}$ and $f \in L_{\text {loc }}^{1}\left(\mathbb{R}^{n}\right)$, we set

$$
\begin{aligned}
A_{\beta}(f)(y, t) & =\sup _{\varphi \in \mathscr{C}_{\beta}}\left|f * \varphi_{t}(y)\right| \\
& =\sup _{\varphi \in \mathscr{C}_{\beta}}\left|\int_{\mathbb{R}^{n}} \varphi_{t}(y-z) f(z) d z\right| .
\end{aligned}
$$

Then we define the intrinsic square function of $f$ (of order $\beta$ ) by the following formula:

$$
\mathcal{S}_{\beta}(f)(x)=\left(\iint_{\Gamma(x)}\left(A_{\beta}(f)(y, t)\right)^{2} \frac{d y d t}{t^{n+1}}\right)^{1 / 2} .
$$

We can also define varying-aperture versions of $\mathcal{S}_{\beta}(f)$ by the formula

$$
\mathcal{S}_{\beta, \gamma}(f)(x)=\left(\iint_{\Gamma_{\gamma}(x)}\left(A_{\beta}(f)(y, t)\right)^{2} \frac{d y d t}{t^{n+1}}\right)^{1 / 2} .
$$

The intrinsic Littlewood-Paley $\mathscr{G}$-function and the intrinsic $\mathscr{G}_{\lambda}^{*}$-function will be given, respectively, by

$$
\begin{aligned}
\mathscr{G}_{\beta}(f)(x) & =\left(\int_{0}^{\infty}\left(A_{\beta}(f)(x, t)\right)^{2} \frac{d t}{t}\right)^{1 / 2}, \\
\mathscr{G}_{\lambda, \beta}^{*}(f)(x)=\left(\iint_{\mathbb{R}_{+}^{n+1}}\left(\frac{t}{t+|x-y|}\right)^{\lambda n}\right. & \left.\times\left(A_{\beta}(f)(y, t)\right)^{2} \frac{d y d t}{t^{n+1}}\right)^{1 / 2}, \quad \lambda>1 .
\end{aligned}
$$

In [8], Wilson showed the following weighted $L^{p}$ boundedness of the intrinsic square functions.

Theorem A. Let $0<\beta \leq 1,1<p<\infty$, and $w \in$ $A_{p}$ (Muckenhoupt weight class). Then there exists a constant $C>0$ independent of $f$ such that

$$
\left\|\mathcal{S}_{\beta}(f)\right\|_{L_{w}^{p}} \leq C\|f\|_{L_{w}^{p}}
$$

Moreover, in [9], Lerner obtained sharp $L_{w}^{p}$ norm inequalities for the intrinsic square functions in terms of the $A_{p}$ characteristic constant of $w$ for all $1<p<\infty$. For further discussions about the boundedness of intrinsic square functions on various function spaces, we refer the readers to [10-17].

Before stating our main results, let us first recall some definitions about the weighted Herz and weak Herz spaces. For more information about these spaces, one can see [18-22] and the references therein. Let $B_{k}=B\left(0,2^{k}\right)=\left\{x \in \mathbb{R}^{n}:|x| \leq 2^{k}\right\}$ and let $C_{k}=B_{k} \backslash B_{k-1}$ for any $k \in \mathbb{Z}$. Denote $\chi_{k}=\chi_{C_{k}}$ for $k \in \mathbb{Z}, \tilde{\chi}_{k}=\chi_{k}$ if $k \in \mathbb{N}$, and $\tilde{\chi}_{0}=\chi_{B_{0}}$, where $\chi_{E}$ is the characteristic function of the set $E$. For any given weight function $w$ on $\mathbb{R}^{n}$ and $0<q<\infty$, we denote by $L_{w}^{q}\left(\mathbb{R}^{n}\right)$ the space of all functions $f$ satisfying

$$
\|f\|_{L_{w}^{q}}=\left(\int_{\mathbb{R}^{n}}|f(x)|^{q} w(x) d x\right)^{1 / q}<\infty .
$$

Definition 1 (see [19]). Let $\alpha \in \mathbb{R}, 0<p$, and $q<\infty$, and let $w_{1}, w_{2}$ be two weight functions on $\mathbb{R}^{n}$.

(a) The homogeneous weighted Herz space $\dot{K}_{q}^{\alpha, p}\left(w_{1}, w_{2}\right)$ is defined by

$$
\begin{aligned}
& \dot{K}_{q}^{\alpha, p}\left(w_{1}, w_{2}\right) \\
& \quad=\left\{f \in L_{\mathrm{loc}}^{q}\left(\mathbb{R}^{n} \backslash\{0\}, w_{2}\right):\|f\|_{\dot{K}_{q}^{\alpha, p}\left(w_{1}, w_{2}\right)}<\infty\right\},
\end{aligned}
$$

where

$$
\|f\|_{\dot{K}_{q}^{\alpha, p}\left(w_{1}, w_{2}\right)}=\left(\sum_{k \in \mathbb{Z}}\left[w_{1}\left(B_{k}\right)\right]^{\alpha p / n}\left\|f \chi_{k}\right\|_{L_{w_{2}}^{q}}^{p}\right)^{1 / p} .
$$

(b) The nonhomogeneous weighted Herz space $K_{q}^{\alpha, p}\left(w_{1}\right.$, $w_{2}$ ) is defined by

$$
\begin{aligned}
& K_{q}^{\alpha, p}\left(w_{1}, w_{2}\right) \\
& \quad=\left\{f \in L_{\text {loc }}^{q}\left(\mathbb{R}^{n}, w_{2}\right):\|f\|_{K_{q}^{\alpha, p}\left(w_{1}, w_{2}\right)}<\infty\right\},
\end{aligned}
$$

where

$$
\|f\|_{K_{q}^{\alpha, p}\left(w_{1}, w_{2}\right)}=\left(\sum_{k=0}^{\infty}\left[w_{1}\left(B_{k}\right)\right]^{\alpha p / n}\left\|f \tilde{\chi}_{k}\right\|_{L_{w_{2}}^{q}}^{p}\right)^{1 / p} .
$$

For any $k \in \mathbb{Z}, \lambda>0$, and any measurable function $f$ on $\mathbb{R}^{n}$, we set $E_{k}(\lambda, f)=\left\{x \in C_{k}:|f(x)|>\lambda\right\}$. Let $\widetilde{E}_{k}(\lambda, f)=$ $E_{k}(\lambda, f)$ for $k \in \mathbb{N}$ and $\widetilde{E}_{0}(\lambda, f)=\{x \in B(0,1):|f(x)|>\lambda\}$.

Definition 2 (see [21]). Let $\alpha \in \mathbb{R}, 0<p$, and $q<\infty$, and let $w_{1}, w_{2}$ be two weight functions on $\mathbb{R}^{n}$.

(c) A measurable function $f(x)$ on $\mathbb{R}^{n}$ is said to belong to the homogeneous weighted weak Herz space $W \dot{K}_{q}^{\alpha, p}\left(w_{1}, w_{2}\right)$ if

$$
\begin{aligned}
&\|f\|_{W \dot{K}_{q}^{\alpha, p}\left(w_{1}, w_{2}\right)} \\
& \quad=\sup _{\lambda>0} \lambda\left(\sum_{k \in \mathbb{Z}}\left[w_{1}\left(B_{k}\right)\right]^{\alpha p / n}\left[w_{2}\left(E_{k}(\lambda, f)\right)\right]^{p / q}\right)^{1 / p}<\infty .
\end{aligned}
$$


(d) A measurable function $f(x)$ on $\mathbb{R}^{n}$ is said to belong to the nonhomogeneous weighted weak Herz space $W K_{q}^{\alpha, p}\left(w_{1}, w_{2}\right)$ if

$$
\begin{aligned}
& \|f\|_{W K_{q}^{\alpha, p}\left(w_{1}, w_{2}\right)} \\
& \quad=\sup _{\lambda>0} \lambda\left(\sum_{k=0}^{\infty}\left[w_{1}\left(B_{k}\right)\right]^{\alpha p / n}\left[w_{2}\left(\widetilde{E}_{k}(\lambda, f)\right)\right]^{p / q}\right)^{1 / p}<\infty .
\end{aligned}
$$

Obviously, if $\alpha=0$, then $\dot{K}_{q}^{0, q}\left(w_{1}, w_{2}\right)=K_{q}^{0, q}\left(w_{1}, w_{2}\right)=$ $L_{w_{2}}^{q}\left(\mathbb{R}^{n}\right)$ for any $0<q<\infty$. We also have $W \dot{K}_{q}^{0, q}\left(w_{1}, w_{2}\right)=$ $W K_{q}^{0, q}\left(w_{1}, w_{2}\right)=W L_{w_{2}}^{q}\left(\mathbb{R}^{n}\right)$ when $\alpha=0$ and $0<q<\infty$, where

$$
\|f\|_{W L_{w}^{q}}=\sup _{\lambda>0} \lambda \cdot w\left(\left\{x \in \mathbb{R}^{n}:|f(x)|>\lambda\right\}\right)^{1 / q}<\infty .
$$

Thus, weighted (weak) Herz spaces are generalizations of the weighted (weak) Lebesgue spaces. The main purpose of this paper is to consider the boundedness of intrinsic square functions on weighted Herz spaces with $A_{p}$ weights. At the extreme case, we will also prove that these operators are bounded from the weighted Herz spaces to the weighted weak Herz spaces. Our main results in the paper are formulated as follows.

Theorem 3. Let $0<\beta \leq 1,0<p<\infty, 1<q<\infty, w_{1} \in A_{q_{1}}$, and $w_{2} \in A_{q_{2}}$. Then $\mathcal{S}_{\beta}$ is bounded on $\dot{K}_{q}^{\alpha, p}\left(w_{1}, w_{2}\right)\left(K_{q}^{\alpha, p}\left(w_{1}\right.\right.$, $\left.w_{2}\right)$ ) provided that $w_{1}$ and $w_{2}$ satisfy either of the following:

(i) $w_{1}=w_{2}, 1 \leq q_{1}=q_{2} \leq q$, and $-n q_{1} / q<\alpha q_{1}<$ $n\left(1-q_{2} / q\right)$;

(ii) $w_{1} \neq w_{2}, 1 \leq q_{1}<\infty, 1 \leq q_{2} \leq q$, and $0<\alpha q_{1}<$ $n\left(1-q_{2} / q\right)$.

Theorem 4. Let $0<\beta \leq 1,0<p \leq 1,1<q<\infty, w_{1} \in A_{q_{1}}$, and $w_{2} \in A_{q_{2}}$. If $1 \leq q_{1}<\infty, 1 \leq q_{2} \leq q$, and $\alpha q_{1}=n(1-$ $\left.q_{2} / q\right)$, then $\mathcal{S}_{\beta}$ is bounded from $\dot{K}_{q}^{\alpha, p}\left(w_{1}, w_{2}\right)\left(K_{q}^{\alpha, p}\left(w_{1}, w_{2}\right)\right)$ into $W \dot{K}_{q}^{\alpha, p}\left(w_{1}, w_{2}\right)\left(W K_{q}^{\alpha, p}\left(w_{1}, w_{2}\right)\right)$.

Theorem 5. Let $0<\beta \leq 1,0<p<\infty, 1<q<\infty, w_{1} \in$ $A_{q_{1}}$, and $w_{2} \in A_{q_{2}}$. If $\lambda>\max \left\{q_{2}, 3\right\}$, then $\mathscr{G}_{\lambda, \beta}^{*}$ is bounded on $\dot{K}_{q}^{\alpha, p}\left(w_{1}, w_{2}\right)\left(K_{q}^{\alpha, p}\left(w_{1}, w_{2}\right)\right)$ provided that $w_{1}$ and $w_{2}$ satisfy either of the following:

(i) $w_{1}=w_{2}, 1 \leq q_{1}=q_{2} \leq q$, and $-n q_{1} / q<\alpha q_{1}<$ $n\left(1-q_{2} / q\right)$;

(ii) $w_{1} \neq w_{2}, 1 \leq q_{1}<\infty, 1 \leq q_{2} \leq q$, and $0<\alpha q_{1}<$ $n\left(1-q_{2} / q\right)$.

Theorem 6. Let $0<\beta \leq 1,0<p \leq 1,1<q<\infty, w_{1} \in$ $A_{q_{1}}$, and $w_{2} \in A_{q_{2}}$. If $1 \leq q_{1}<\infty, 1 \leq q_{2} \leq q$, $\alpha q_{1}=n\left(1-q_{2} / q\right)$, and $\lambda>\max \left\{q_{2}, 3\right\}$, then $\mathscr{G}_{\lambda, \beta}^{*}$ is bounded from $\dot{K}_{q}^{\alpha, p}\left(w_{1}, w_{2}\right)\left(K_{q}^{\alpha, p}\left(w_{1}, w_{2}\right)\right)$ into $W \dot{K}_{q}^{\alpha, p}\left(w_{1}\right.$, $\left.w_{2}\right)\left(W K_{q}^{\alpha, p}\left(w_{1}, w_{2}\right)\right)$.
In [7], Wilson also showed that for any $0<\beta \leq 1$, the functions $\mathcal{S}_{\beta}(f)(x)$ and $\mathscr{G}_{\beta}(f)(x)$ are pointwise comparable, with comparability constants depending only on $\beta$ and $n$. Thus, as direct consequences of Theorems 3 and 4, we obtain the following.

Corollary 7. Let $0<\beta \leq 1,0<p<\infty, 1<q<\infty$, $w_{1} \in A_{q_{1}}$, and $w_{2} \in A_{q_{2}}$. Then $\mathscr{G}_{\beta}$ is bounded on $\dot{K}_{q}^{\alpha, p}\left(w_{1}\right.$, $\left.w_{2}\right)\left(K_{q}^{\alpha, p}\left(w_{1}, w_{2}\right)\right)$ provided that $w_{1}$ and $w_{2}$ satisfy either of the following:

(i) $w_{1}=w_{2}, 1 \leq q_{1}=q_{2} \leq q$, and $-n q_{1} / q<\alpha q_{1}<$ $n\left(1-q_{2} / q\right)$;

(ii) $w_{1} \neq w_{2}, 1 \leq q_{1}<\infty, 1 \leq q_{2} \leq q$, and $0<\alpha q_{1}<$ $n\left(1-q_{2} / q\right)$.

Corollary 8. Let $0<\beta \leq 1,0<p \leq 1,1<q<\infty, w_{1} \in A_{q_{1}}$, and $w_{2} \in A_{q_{2}}$. If $1 \leq q_{1}<\infty, 1 \leq q_{2} \leq q$, and $\alpha q_{1}=n(1-$ $\left.q_{2} / q\right)$, then $\mathscr{G}_{\beta}$ is bounded from $\dot{K}_{q}^{\alpha, p}\left(w_{1}, w_{2}\right)\left(K_{q}^{\alpha, p}\left(w_{1}, w_{2}\right)\right)$ into $W \dot{K}_{q}^{\alpha, p}\left(w_{1}, w_{2}\right)\left(W K_{q}^{\alpha, p}\left(w_{1}, w_{2}\right)\right)$.

\section{2. $A_{p}$ Weights}

The classical $A_{p}$ weight theory was first introduced by Muckenhoupt in the study of weighted $L^{p}$ boundedness of Hardy-Littlewood maximal functions in [23]. A weight $w$ is a nonnegative, locally integrable function on $\mathbb{R}^{n}, B=B\left(x_{0}, r_{B}\right)$ that denotes the ball with the center $x_{0}$ and radius $r_{B}$. For any ball $B$ and $\lambda>0, \lambda B$ denotes the ball concentric with $B$ whose radius is $\lambda$ times as long. For a given weight function $w$ and a measurable set $E$, we also denote the Lebesgue measure of $E$ by $|E|$ and set weighted measure $w(E)=\int_{E} w(x) d x$. We say that $w$ is in the Muckenhoupt class $A_{p}$ with $1<p<\infty$, if there exists a constant $C>0$ such that for every ball $B \subseteq \mathbb{R}^{n}$,

$$
\left(\frac{1}{|B|} \int_{B} w(x) d x\right)\left(\frac{1}{|B|} \int_{B} w(x)^{-1 /(p-1)} d x\right)^{p-1} \leq C .
$$

For the endpoint case $p=1, w \in A_{1}$, if

$$
\begin{array}{r}
\frac{1}{|B|} \int_{B} w(x) d x \leq C \cdot \text { ess } \inf _{x \in B} w(x) \\
\text { for every ball } B \subseteq \mathbb{R}^{n},
\end{array}
$$

where $C$ is a positive constant which is independent of the choice of $B$. The smallest value of $C$ such that the above inequalities hold is called the $A_{p}$ characteristic constant of $w$ and denoted by $[w]_{A_{p}}$. If there exist two constants $r>1$ and $C>0$ such that the following reverse Hölder inequality holds:

$$
\left(\frac{1}{|B|} \int_{B} w(x)^{r} d x\right)^{1 / r} \leq C\left(\frac{1}{|B|} \int_{B} w(x) d x\right)
$$

for every ball $B \subseteq \mathbb{R}^{n}$,

then we say that $w$ satisfies the reverse Hölder condition of order $r$ and write $w \in R H_{r}$. It is well known that if $w \in A_{p}$ 
with $1 \leq p<\infty$, then $w \in A_{q}$ for all $q>p$. Moreover, if $w \in A_{p}$ with $1 \leq p<\infty$, then there exists $r>1$ such that $w \in R H_{r}$.

The following properties for $A_{p}$ weights will be repeatedly used in this paper.

Lemma 9 (see [24]). Let $w \in A_{p}$ with $p \geq 1$. Then, for any ball $B$, there exists an absolute constant $C>0$ such that

$$
w(2 B) \leq C w(B) .
$$

In general, for any $\lambda>1$, one has

$$
w(\lambda B) \leq C \cdot \lambda^{n p} w(B),
$$

where $C$ does not depend on $B$ nor on $\lambda$.

Lemma 10 (see $[1,24]$ ). Let $w \in A_{p} \cap R H_{r}, p \geq 1$, and $r>1$. Then there exist constants $C_{1}, C_{2}>0$ such that

$$
C_{1}\left(\frac{|E|}{|B|}\right)^{p} \leq \frac{w(E)}{w(B)} \leq C_{2}\left(\frac{|E|}{|B|}\right)^{(r-1) / r}
$$

for any measurable subset $E$ of a ball $B$.

Throughout this paper, $C$ always denotes a positive constant which is independent of the main parameters involved but may vary from line to line.

\section{Proofs of Theorems 3 and 4}

Proof of Theorem 3. We only need to show the theorem for the homogeneous case because the proof of the nonhomogeneous result is similar and so is omitted here. Let $f \in$ $\dot{K}_{q}^{\alpha, p}\left(w_{1}, w_{2}\right)$. Following [25], for any $k \in \mathbb{Z}$, we decompose $f(x)$ as

$$
\begin{aligned}
f(x)= & f(x) \chi_{\left\{2^{k-2}<|x| \leq 2^{k+1}\right\}}(x) \\
& +f(x) \chi_{\left\{|x| \leq 2^{k-2}\right\}}(x)+f(x) \chi_{\left\{|x|>2^{k+1}\right\}}(x) \\
= & f_{1}(x)+f_{2}(x)+f_{3}(x) .
\end{aligned}
$$

Since $\mathcal{S}_{\beta}(0<\beta \leq 1)$ is a sublinear operator, then we can write

$$
\begin{aligned}
& \left\|\mathcal{S}_{\beta}(f)\right\|_{\dot{K}_{q}^{\alpha, p}\left(w_{1}, w_{2}\right)}^{p} \\
& \quad=\sum_{k \in \mathbb{Z}}\left[w_{1}\left(B_{k}\right)\right]^{\alpha p / n}\left\|\mathcal{S}_{\beta}(f) \chi_{k}\right\|_{L_{w_{2}}^{q}}^{p} \\
& \quad \leq C \sum_{i=1}^{3} \sum_{k \in \mathbb{Z}}\left[w_{1}\left(B_{k}\right)\right]^{\alpha p / n}\left\|\mathcal{S}_{\beta}\left(f_{i}\right) \chi_{k}\right\|_{L_{w_{2}}^{q}}^{p} \\
& \quad=I_{1}+I_{2}+I_{3} .
\end{aligned}
$$

Since $w_{2} \in A_{q_{2}}$ and $1 \leq q_{2} \leq q$, then $w_{2} \in A_{q}$. By Theorem A and Lemma 9 , we have

$$
\begin{aligned}
I_{1} & \leq C \sum_{k \in \mathbb{Z}}\left[w_{1}\left(B_{k}\right)\right]^{\alpha p / n}\left\|f_{1}\right\|_{L_{w_{2}}^{q}}^{p} \\
& \leq C \sum_{k \in \mathbb{Z}}\left[w_{1}\left(B_{k}\right)\right]^{\alpha p / n}\left\|f \chi_{k}\right\|_{L_{w_{2}}^{q}}^{p} \\
& \leq C\|f\|_{\dot{K}_{q}^{\alpha, p}\left(w_{1}, w_{2}\right)}^{p} .
\end{aligned}
$$

For the term $I_{2}$, we first use Minkowski's inequality to derive

$$
I_{2} \leq C \sum_{k \in \mathbb{Z}}\left[w_{1}\left(B_{k}\right)\right]^{\alpha p / n}\left(\sum_{\ell=-\infty}^{k-2}\left\|\mathcal{S}_{\beta}\left(f \chi_{\ell}\right) \chi_{k}\right\|_{L_{w_{2}}^{q}}\right)^{p} .
$$

For any $\varphi \in \mathscr{C}_{\beta}, 0<\beta \leq 1$, and $(y, t) \in \Gamma(x)$, we have

$$
\begin{aligned}
\left|\left(f \chi_{\ell}\right) * \varphi_{t}(y)\right| & =\left|\int_{2^{\ell-1}<|z| \leq 2^{\ell}} \varphi_{t}(y-z) f(z) d z\right| \\
& \leq C \cdot t^{-n} \int_{\left\{2^{\ell-1}<|z| \leq 2^{\ell}\right\} \cap\{z:|y-z| \leq t\}}|f(z)| d z .
\end{aligned}
$$

For any $x \in C_{k},(y, t) \in \Gamma(x)$, and $z \in\left\{2^{\ell-1}<|z| \leq 2^{\ell}\right\} \cap$ $B(y, t)$ with $\ell \leq k-2$, by a direct computation, we can easily see that

$$
2 t \geq|x-y|+|y-z| \geq|x-z| \geq|x|-|z| \geq \frac{|x|}{2} .
$$

Thus, by using inequality (31) and Minkowski’s inequality, we deduce

$$
\begin{aligned}
& \left|\mathcal{S}_{\beta}\left(f \chi_{\ell}\right)(x)\right| \\
& \quad=\left(\iint_{\Gamma(x)}\left(\sup _{\varphi \in \mathscr{C}_{\beta}}\left|\left(f \chi_{\ell}\right) * \varphi_{t}(y)\right|\right)^{2} \frac{d y d t}{t^{n+1}}\right)^{1 / 2} \\
& \quad \leq C\left(\int_{|x| / 4}^{\infty} \int_{|x-y|<t}\left|t^{-n} \int_{2^{\ell-1}<|z| \leq 2^{\ell}}\right| f(z)|d z|^{2} \frac{d y d t}{t^{n+1}}\right)^{1 / 2} \\
& \quad \leq C\left(\int_{2^{\ell-1}<|z| \leq 2^{\ell}}|f(z)| d z\right)\left(\int_{|x| / 4}^{\infty} \frac{d t}{t^{2 n+1}}\right)^{1 / 2} \\
& \quad \leq C \cdot \frac{1}{|x|^{n}}\left(\int_{2^{\ell-1}<|z| \leq 2^{\ell}}|f(z)| d z\right) .
\end{aligned}
$$


Denote the conjugate exponent of $q>1$ by $q^{\prime}=q /(q-1)$. Applying Hölder's inequality and the $A_{q}$ condition, we can deduce that

$$
\begin{aligned}
& \int_{2^{\ell-1}<|z| \leq 2^{\ell}}|f(z)| d z \\
& \leq\left(\int_{2^{\ell-1}<|z| \leq 2^{\ell}}|f(z)|^{q} w_{2}(z) d z\right)^{1 / q} \\
& \quad \times\left(\int_{2^{\ell-1}<|z| \leq 2^{\ell}} w_{2}(z)^{-q^{\prime} / q} d z\right)^{1 / q^{\prime}} \\
& \leq C \cdot\left|B_{\ell}\right|\left[w_{2}\left(B_{\ell}\right)\right]^{-1 / q}\left\|f \chi_{\ell}\right\|_{L_{w_{2}}^{q}} .
\end{aligned}
$$

Substituting inequality (34) into (33), we thus obtain

$$
\begin{aligned}
I_{2} \leq & C \sum_{k \in \mathbb{Z}}\left[w_{1}\left(B_{k}\right)\right]^{\alpha p / n} \\
& \times\left(\sum_{\ell=-\infty}^{k-2}\left\{\int_{2^{k-1}<|x| \leq 2^{k}}\left|\mathcal{S}_{\beta}\left(f \chi_{\ell}\right)(x)\right|^{q} w_{2}(x) d x\right\}^{1 / q}\right)^{p} \\
\leq & C \sum_{k \in \mathbb{Z}}\left[w_{1}\left(B_{k}\right)\right]^{\alpha p / n} \\
& \times\left(\sum_{\ell=-\infty}^{k-2}\left|B_{\ell}\right|\left[w_{2}\left(B_{\ell}\right)\right]^{-1 / q}\left\|f \chi_{\ell}\right\|_{L_{w_{2}}^{q}}\right. \\
\leq & \left.C \sum_{k \in \mathbb{Z}}\left[w_{1}\left(B_{k}\right)\right]^{\alpha p / n}\left\{\int_{2^{k-1}<|x| \leq 2^{k}} \frac{w_{2}(x)}{|x|^{n q}} d x\right\}^{1 / q}\right)^{p} \\
& \times\left(\sum_{\ell=-\infty}^{k-2} \frac{\left|B_{\ell}\right|}{\left|B_{k}\right|} \cdot \frac{\left[w_{2}\left(B_{k}\right)\right]^{1 / q}}{\left[w_{2}\left(B_{\ell}\right)\right]^{1 / q}}\left\|f \chi_{\ell}\right\|_{L_{w_{2}}^{q}}\right)^{p}
\end{aligned}
$$

Here, we will consider two cases. For the case of $0<p \leq$ 1 , using the well-known inequality $\left(\sum_{\ell}\left|a_{\ell}\right|\right)^{p} \leq \sum_{\ell}\left|a_{\ell}\right|^{p}$ and changing the order of summation, we find that

$$
\begin{aligned}
I_{2} \leq & C \sum_{\ell \in \mathbb{Z}}\left[w_{1}\left(B_{\ell}\right)\right]^{\alpha p / n}\left\|f \chi_{\ell}\right\|_{L_{w_{2}}^{q}}^{p} \\
& \times\left(\sum_{k=\ell+2}^{\infty} \frac{\left|B_{\ell}\right|^{p}}{\left|B_{k}\right|^{p}} \cdot \frac{\left[w_{2}\left(B_{k}\right)\right]^{p / q}}{\left[w_{2}\left(B_{\ell}\right)\right]^{p / q}} \cdot \frac{\left[w_{1}\left(B_{k}\right)\right]^{\alpha p / n}}{\left[w_{1}\left(B_{\ell}\right)\right]^{\alpha p / n}}\right) .
\end{aligned}
$$

Moreover, it follows immediately from Lemma 9 that

$$
\begin{aligned}
I_{2} \leq & C \sum_{\ell \in \mathbb{Z}}\left[w_{1}\left(B_{\ell}\right)\right]^{\alpha p / n}\left\|f \chi_{\ell}\right\|_{L_{w_{2}}^{q}}^{p} \\
& \times\left(\sum_{k=\ell+2}^{\infty} \frac{\left|B_{\ell}\right|^{p}}{\left|B_{k}\right|^{p}} \cdot \frac{\left[w_{2}\left(B_{k}\right)\right]^{p / q}}{\left[w_{2}\left(B_{\ell+2}\right)\right]^{p / q}} \cdot \frac{\left[w_{1}\left(B_{k}\right)\right]^{\alpha p / n}}{\left[w_{1}\left(B_{\ell+2}\right)\right]^{\alpha p / n}}\right) .
\end{aligned}
$$

Since $B_{k} \supseteq B_{\ell+2}$ when $k \geq \ell+2$ and $w_{i} \in A_{q_{i}}$ for $i=1,2$, by Lemma 10 , we can get

$$
\frac{w_{i}\left(B_{k}\right)}{w_{i}\left(B_{\ell+2}\right)} \leq C\left(\frac{\left|B_{k}\right|}{\left|B_{\ell+2}\right|}\right)^{q_{i}}, \quad \text { for } i=1 \text { and } 2 \text {. }
$$

Therefore

$$
\begin{aligned}
I_{2} \leq & C \sum_{\ell \in \mathbb{Z}}\left[w_{1}\left(B_{\ell}\right)\right]^{\alpha p / n}\left\|f \chi_{\ell}\right\|_{L_{w_{2}}^{q}}^{p} \\
& \times\left(\sum_{k=\ell+2}^{\infty}\left[\frac{\left|B_{\ell+2}\right|}{\left|B_{k}\right|}\right]^{p-\alpha q_{1} p / n-q_{2} p / q}\right) \\
\leq & C \sum_{\ell \in \mathbb{Z}}\left[w_{1}\left(B_{\ell}\right)\right]^{\alpha p / n}\left\|f \chi_{\ell}\right\|_{L_{w_{2}}^{q}}^{p} \\
& \times\left(\sum_{k=0}^{\infty} 2^{-k n\left(p-\alpha q_{1} p / n-q_{2} p / q\right)}\right) \\
\leq & C \sum_{\ell \in \mathbb{Z}}\left[w_{1}\left(B_{\ell}\right)\right]^{\alpha p / n}\left\|f \chi_{\ell}\right\|_{L_{w_{2}}^{q}}^{p},
\end{aligned}
$$

where the last inequality holds since $\alpha q_{1}<n\left(1-q_{2} / q\right)$. On the other hand, for the case of $1<p<\infty$, we will use Hölder's inequality to obtain

$$
\begin{gathered}
\left(\sum_{\ell=-\infty}^{k-2} \frac{\left|B_{\ell}\right|}{\left|B_{k}\right|} \cdot \frac{\left[w_{2}\left(B_{k}\right)\right]^{1 / q}}{\left[w_{2}\left(B_{\ell}\right)\right]^{1 / q}} \cdot\left[w_{1}\left(B_{k}\right)\right]^{\alpha / n}\left\|f \chi_{\ell}\right\|_{L_{w_{2}}^{q}}^{p}\right)^{p} \\
\leq\left(\sum_{\ell=-\infty}^{k-2}\left[w_{1}\left(B_{\ell}\right)\right]^{\alpha p / n}\left\|f \chi_{\ell}\right\|_{L_{w_{2}}^{q}}^{p}\right. \\
\left.\times \frac{\left|B_{\ell}\right|^{p / 2}}{\left|B_{k}\right|^{p / 2}} \cdot \frac{\left[w_{2}\left(B_{k}\right)\right]^{p / 2 q}}{\left[w_{2}\left(B_{\ell}\right)\right]^{p / 2 q}} \cdot \frac{\left[w_{1}\left(B_{k}\right)\right]^{\alpha p / 2 n}}{\left[w_{1}\left(B_{\ell}\right)\right]^{\alpha p / 2 n}}\right) \\
\times\left(\sum_{\ell=-\infty\left|B_{k}\right|^{p^{\prime} / 2}}^{k-2} \frac{\left|B_{\ell}\right|^{p^{\prime} / 2}}{\left[w_{2}\left(B_{k}\right)\right]^{p^{\prime} / 2 q}}\right. \\
\left.\times \frac{\left.\left[B_{\ell}\right)\right]^{p^{\prime} / 2 q}}{\left[w_{1}\left(B_{k}\right)\right]^{\alpha p^{\prime} / 2 n}}\right]^{\alpha p^{\prime} / 2 n}
\end{gathered}
$$

Using the same arguments as above, we can also prove the following estimates under the assumption that $\alpha q_{1}<n(1-$ $\left.q_{2} / q\right)$ :

$$
\begin{aligned}
& \sum_{k=\ell+2}^{\infty} \frac{\left|B_{\ell}\right|^{p / 2}}{\left|B_{k}\right|^{p / 2}} \cdot \frac{\left[w_{2}\left(B_{k}\right)\right]^{p / 2 q}}{\left[w_{2}\left(B_{\ell}\right)\right]^{p / 2 q}} \cdot \frac{\left[w_{1}\left(B_{k}\right)\right]^{\alpha p / 2 n}}{\left[w_{1}\left(B_{\ell}\right)\right]^{\alpha p / 2 n}} \leq C, \\
& \sum_{\ell=-\infty}^{k-2} \frac{\left|B_{\ell}\right|^{p^{\prime} / 2}}{\left|B_{k}\right|^{p^{\prime} / 2}} \cdot \frac{\left[w_{2}\left(B_{k}\right)\right]^{p^{\prime} / 2 q}}{\left[w_{2}\left(B_{\ell}\right)\right]^{p^{\prime} / 2 q}} \cdot \frac{\left[w_{1}\left(B_{k}\right)\right]^{\alpha p^{\prime} / 2 n}}{\left[w_{1}\left(B_{\ell}\right)\right]^{\alpha p^{\prime} / 2 n}} \leq C .
\end{aligned}
$$


Hence

$$
\begin{aligned}
I_{2} \leq & C \sum_{k \in \mathbb{Z}}\left(\sum_{\ell=-\infty}^{k-2}\left[w_{1}\left(B_{\ell}\right)\right]^{\alpha p / n}\left\|f \chi_{\ell}\right\|_{L_{w_{2}}^{q}}^{p}\right. \\
& \left.\times \frac{\left|B_{\ell}\right|^{p / 2}}{\left|B_{k}\right|^{p / 2}} \cdot \frac{\left[w_{2}\left(B_{k}\right)\right]^{p / 2 q}}{\left[w_{2}\left(B_{\ell}\right)\right]^{p / 2 q}} \cdot \frac{\left[w_{1}\left(B_{k}\right)\right]^{\alpha p / 2 n}}{\left[w_{1}\left(B_{\ell}\right)\right]^{\alpha p / 2 n}}\right) \\
\leq & C \sum_{\ell \in \mathbb{Z}}\left[w_{1}\left(B_{\ell}\right)\right]^{\alpha p / n}\left\|f \chi_{\ell}\right\|_{L_{w_{2}}^{q}}^{p} \\
& \times\left(\sum_{k=\ell+2}^{\infty} \frac{\left|B_{\ell}\right|^{p / 2}}{\left[\left.B_{k}\right|^{p / 2}\right.} \cdot \frac{\left[w_{2}\left(B_{k}\right)\right]^{p / 2 q}}{\left[w_{2}\left(B_{\ell}\right)\right]^{p / 2 q}} \cdot \frac{\left[w_{1}\left(B_{k}\right)\right]^{\alpha p / 2 n}}{\left[w_{1}\left(B_{\ell}\right)\right]^{\alpha p / 2 n}}\right) \\
\leq & C \sum_{\ell \in \mathbb{Z}}\left[w_{1}\left(B_{\ell}\right)\right]^{\alpha p / n}\left\|f \chi_{\ell}\right\|_{L_{w_{2}}^{q}}^{p} \cdot
\end{aligned}
$$

Summarizing the above estimates for the term $I_{2}$, we obtain that for every $0<p<\infty$,

$$
I_{2} \leq C \sum_{\ell \in \mathbb{Z}}\left[w_{1}\left(B_{\ell}\right)\right]^{\alpha p / n}\left\|f \chi_{\ell}\right\|_{L_{w_{2}}^{q}}^{p} \leq C\|f\|_{\dot{K}_{q}^{\alpha, p}\left(w_{1}, w_{2}\right)}^{p}
$$

Let us now turn to estimate the last term $I_{3}$. In this case, for any $x \in C_{k},(y, t) \in \Gamma(x)$, and $z \in\left\{2^{\ell-1}<|z| \leq 2^{\ell}\right\} \cap B(y, t)$ with $\ell \geq k+2$, it is easy to check that

$$
2 t \geq|x-y|+|y-z| \geq|x-z| \geq|z|-|x| \geq \frac{|z|}{2} .
$$

Then it follows from inequality (31) and Minkowski's inequality that

$$
\begin{aligned}
& \left|\mathcal{S}_{\beta}\left(f \chi_{\ell}\right)(x)\right| \\
& \quad \leq C\left(\int_{|z| / 4}^{\infty} \int_{|x-y|<t}\left|t^{-n} \int_{2^{\ell-1}<|z| \leq 2^{\ell}}\right| f(z)|d z|^{2} \frac{d y d t}{t^{n+1}}\right)^{1 / 2} \\
& \quad \leq C\left(\int_{2^{\ell-1}<|z| \leq 2^{\ell}}|f(z)| d z\right)\left(\int_{|z| / 4}^{\infty} \frac{d t}{t^{2 n+1}}\right)^{1 / 2} \\
& \quad \leq C\left(\int_{2^{\ell-1}<|z| \leq 2^{\ell}} \frac{|f(z)|}{|z|^{n}} d z\right) .
\end{aligned}
$$

This estimate together with (34) implies that

$$
\begin{aligned}
\left|\mathcal{S}_{\beta}\left(f \chi_{\ell}\right)(x)\right| & \leq C \cdot \frac{1}{\left|B_{\ell}\right|}\left(\int_{2^{\ell-1}<|z| \leq 2^{\ell}}|f(z)| d z\right) \\
& \leq C \cdot\left[w_{2}\left(B_{\ell}\right)\right]^{-1 / q}\left\|f \chi_{\ell}\right\|_{L_{w_{2}}^{q} .}
\end{aligned}
$$

Hence

$$
\begin{aligned}
I_{3} \leq & C \sum_{k \in \mathbb{Z}}\left[w_{1}\left(B_{k}\right)\right]^{\alpha p / n} \\
& \times\left(\sum_{\ell=k+2}^{\infty}\left\{\int_{2^{k-1}<|x| \leq 2^{k}}\left|\mathcal{S}_{\beta}\left(f \chi_{\ell}\right)(x)\right|^{q} w_{2}(x) d x\right\}^{1 / q}\right)^{p} \\
\leq & C \sum_{k \in \mathbb{Z}}\left[w_{1}\left(B_{k}\right)\right]^{\alpha p / n}\left(\sum_{\ell=k+2}^{\infty}\left[w_{2}\left(B_{\ell}\right)\right]^{-1 / q}\left\|f \chi_{\ell}\right\|_{L_{w_{2}}^{q}}\right. \\
\leq & C \sum_{k \in \mathbb{Z}}\left[w_{1}\left(B_{k}\right)\right]^{\alpha p / n}\left(\sum_{\ell=k+2}^{\infty} \frac{\left[w_{2}\left(B_{k}\right)\right]^{1 / q}}{\left[w_{2}\left(B_{\ell}\right)\right]^{1 / q}}\left\|f \chi_{\ell}\right\|_{L_{w_{2}}^{q}}\right)^{p} .
\end{aligned}
$$

Now we will consider the following two cases again. For the case of $0<p \leq 1$, by using the inequality $\left(\sum_{\ell}\left|a_{\ell}\right|\right)^{p} \leq \sum_{\ell}\left|a_{\ell}\right|^{p}$ and changing the order of summation, we obtain

$$
\begin{aligned}
I_{3} \leq C \sum_{\ell \in \mathbb{Z}}\left[w_{1}\left(B_{\ell}\right)\right]^{\alpha p / n}\left\|f \chi_{\ell}\right\|_{L_{w_{2}}^{q}}^{p} \\
\times\left(\sum_{k=-\infty}^{\ell-2} \frac{\left[w_{2}\left(B_{k}\right)\right]^{p / q}}{\left[w_{2}\left(B_{\ell}\right)\right]^{p / q}} \cdot \frac{\left[w_{1}\left(B_{k}\right)\right]^{\alpha p / n}}{\left[w_{1}\left(B_{\ell}\right)\right]^{\alpha p / n}}\right) \\
\leq C \sum_{\ell \in \mathbb{Z}}\left[w_{1}\left(B_{\ell}\right)\right]^{\alpha p / n}\left\|f \chi_{\ell}\right\|_{L_{w_{2}}^{q}}^{p} \\
\times\left(\sum_{k=-\infty}^{\ell-2} \frac{\left[w_{2}\left(B_{k}\right)\right]^{p / q}}{\left[w_{2}\left(B_{\ell-2}\right)\right]^{p / q}} \cdot \frac{\left[w_{1}\left(B_{k}\right)\right]^{\alpha p / n}}{\left[w_{1}\left(B_{\ell-2}\right)\right]^{\alpha p / n}}\right) .
\end{aligned}
$$

Since $w_{i} \in A_{q_{i}}$, then there exists $r_{i}>1$ such that $w_{i} \in R H_{r_{i}}$ for $i=1,2$. Thus, by Lemma 10 again, we can get

$$
\frac{w_{i}\left(B_{k}\right)}{w_{i}\left(B_{\ell-2}\right)} \leq C\left(\frac{\left|B_{k}\right|}{\left|B_{\ell-2}\right|}\right)^{\delta_{i}}, \quad \text { for } i=1 \text { and } 2,
$$

where $\delta_{i}=\left(r_{i}-1\right) / r_{i}>0$. Therefore, we have

$$
\begin{aligned}
& I_{3} \leq C \sum_{\ell \in \mathbb{Z}}\left[w_{1}\left(B_{\ell}\right)\right]^{\alpha p / n}\left\|f \chi_{\ell}\right\|_{L_{w_{2}}^{q}}^{p} \\
& \times\left(\sum_{k=-\infty}^{\ell-2}\left[\frac{\left|B_{k}\right|}{\left|B_{\ell-2}\right|}\right]^{\alpha \delta_{1} p / n+\delta_{2} p / q}\right) \\
& \leq C \sum_{\ell \in \mathbb{Z}}\left[w_{1}\left(B_{\ell}\right)\right]^{\alpha p / n}\left\|f \chi_{\ell}\right\|_{L_{w_{2}}^{q}}^{p} \\
& \times\left(\sum_{k=-\infty}^{0} 2^{k n\left(\alpha \delta_{1} p / n+\delta_{2} p / q\right)}\right) \\
& \leq C \sum_{\ell \in \mathbb{Z}}\left[w_{1}\left(B_{\ell}\right)\right]^{\alpha p / n}\left\|f \chi_{\ell}\right\|_{L_{w_{2}}^{q}}^{p},
\end{aligned}
$$

where in the last inequality we have used the fact that $\alpha \delta_{1} p / n+\delta_{2} p / q>0$ under our assumption (i) or (ii). 
On the other hand, for the case of $1<p<\infty$, an application of Hölder's inequality gives us that

$$
\begin{gathered}
\left(\sum_{\ell=k+2}^{\infty} \frac{\left[w_{2}\left(B_{k}\right)\right]^{1 / q}}{\left[w_{2}\left(B_{\ell}\right)\right]^{1 / q}} \cdot\left[w_{1}\left(B_{k}\right)\right]^{\alpha / n}\left\|f \chi_{\ell}\right\|_{L_{w_{2}}^{q}}\right)^{p} \\
\leq\left(\sum_{\ell=k+2}^{\infty}\left[w_{1}\left(B_{\ell}\right)\right]^{\alpha p / n}\left\|f \chi_{\ell}\right\|_{L_{w_{2}}^{q}}^{p}\right. \\
\left.\cdot \frac{\left[w_{2}\left(B_{k}\right)\right]^{p / 2 q}}{\left[w_{2}\left(B_{\ell}\right)\right]^{p / 2 q}} \cdot \frac{\left[w_{1}\left(B_{k}\right)\right]^{\alpha p / 2 n}}{\left[w_{1}\left(B_{\ell}\right)\right]^{\alpha p / 2 n}}\right) \\
\times\left(\sum_{\ell=k+2}^{\infty} \frac{\left[w_{2}\left(B_{k}\right)\right]^{p^{\prime} / 2 q}}{\left.\left[B_{\ell}\right)\right]^{p^{\prime} / 2 q}} \cdot \frac{\left[w_{1}\left(B_{k}\right)\right]^{\alpha p^{\prime} / 2 n}}{\left[w_{1}\left(B_{\ell}\right)\right]^{\alpha p^{\prime} / 2 n}}\right)^{p / p^{\prime}} .
\end{gathered}
$$

By using the same arguments as for $I_{3}$, we are able to prove that the following two series are convergent by an absolute constant under the assumption (i) or (ii):

$$
\begin{aligned}
& \sum_{k=-\infty}^{\ell-2} \frac{\left[w_{2}\left(B_{k}\right)\right]^{p / 2 q}}{\left[w_{2}\left(B_{\ell}\right)\right]^{p / 2 q}} \cdot \frac{\left[w_{1}\left(B_{k}\right)\right]^{\alpha p / 2 n}}{\left[w_{1}\left(B_{\ell}\right)\right]^{\alpha p / 2 n}} \leq C, \\
& \sum_{\ell=k+2}^{\infty} \frac{\left[w_{2}\left(B_{k}\right)\right]^{p^{\prime} / 2 q}}{\left[w_{2}\left(B_{\ell}\right)\right]^{p^{\prime} / 2 q}} \cdot \frac{\left[w_{1}\left(B_{k}\right)\right]^{\alpha p^{\prime} / 2 n}}{\left[w_{1}\left(B_{\ell}\right)\right]^{\alpha p^{\prime} / 2 n}} \leq C .
\end{aligned}
$$

Consequently

$$
\begin{gathered}
I_{3} \leq C \sum_{k \in \mathbb{Z}}\left(\sum_{\ell=k+2}^{\infty}\left[w_{1}\left(B_{\ell}\right)\right]^{\alpha p / n}\left\|f \chi_{\ell}\right\|_{L_{w_{2}}^{q}}^{p}\right. \\
\left.\cdot \frac{\left[w_{2}\left(B_{k}\right)\right]^{p / 2 q}}{\left[w_{2}\left(B_{\ell}\right)\right]^{p / 2 q}} \cdot \frac{\left[w_{1}\left(B_{k}\right)\right]^{\alpha p / 2 n}}{\left[w_{1}\left(B_{\ell}\right)\right]^{\alpha p / 2 n}}\right) \\
\leq C \sum_{\ell \in \mathbb{Z}}\left[w_{1}\left(B_{\ell}\right)\right]^{\alpha / n}\left\|f \chi_{\ell}\right\|_{L_{w_{2}}^{q}}^{p} \\
\times\left(\sum_{k=-\infty}^{\ell-2} \frac{\left[w_{2}\left(B_{k}\right)\right]^{p / 2 q}}{\left[w_{2}\left(B_{\ell}\right)\right]^{p / 2 q}} \cdot \frac{\left[w_{1}\left(B_{k}\right)\right]^{\alpha p / 2 n}}{\left[w_{1}\left(B_{\ell}\right)\right]^{\alpha p / 2 n}}\right) \\
\leq C \sum_{\ell \in \mathbb{Z}}\left[w_{1}\left(B_{\ell}\right)\right]^{\alpha / n / n}\left\|f \chi_{\ell}\right\|_{L_{w_{2}}^{q}}^{p} \cdot
\end{gathered}
$$

From the above discussions for the term $I_{3}$, we know that, for any $0<p<\infty$,

$$
I_{3} \leq C \sum_{\ell \in \mathbb{Z}}\left[w_{1}\left(B_{\ell}\right)\right]^{\alpha p / n}\left\|f \chi_{\ell}\right\|_{L_{w_{2}}^{q}}^{p} \leq C\|f\|_{\dot{K}_{q}^{\alpha, p}\left(w_{1}, w_{2}\right)}^{p} .
$$

Summing up the above estimates for $I_{1}, I_{2}$, and $I_{3}$, we complete the proof of Theorem 3 .
Proof of Theorem 4. Let $f \in \dot{K}_{q}^{\alpha, p}\left(w_{1}, w_{2}\right)$. For any $k \in \mathbb{Z}$, as in the proof of Theorem 3, we will split $f(x)$ into three parts:

$$
\begin{aligned}
f(x)= & f(x) \chi_{\left\{2^{k-2}<|x| \leq 2^{k+1}\right\}}(x)+f(x) \chi_{\left\{|x| \leq 2^{k-2}\right\}}(x) \\
& +f(x) \chi_{\left\{|x|>2^{k+1}\right\}}(x) \\
= & f_{1}(x)+f_{2}(x)+f_{3}(x) .
\end{aligned}
$$

Then, for any given $\lambda>0$, we have

$$
\begin{aligned}
& \lambda^{p} \cdot \sum_{k \in \mathbb{Z}}\left[w_{1}\left(B_{k}\right)\right]^{\alpha p / n} w_{2}\left(\left\{x \in C_{k}:\left|\mathcal{S}_{\beta}(f)(x)\right|>\lambda\right\}\right)^{p / q} \\
& \leq \sum_{i=1}^{3} \lambda^{p} \cdot \sum_{k \in \mathbb{Z}}\left[w_{1}\left(B_{k}\right)\right]^{\alpha p / n} \\
& \quad \times w_{2}\left(\left\{x \in C_{k}:\left|\mathcal{S}_{\beta}\left(f_{i}\right)(x)\right|>\frac{\lambda}{3}\right\}\right)^{p / q} \\
& =I_{1}^{\prime}+I_{2}^{\prime}+I_{3}^{\prime} .
\end{aligned}
$$

Applying Chebyshev's inequality, Theorem A, and Lemma 9, we obtain

$$
\begin{aligned}
I_{1}^{\prime} & \leq \lambda^{p} \cdot \sum_{k \in \mathbb{Z}}\left[w_{1}\left(B_{k}\right)\right]^{\alpha p / n}\left(\frac{3^{q}}{\lambda^{q}}\left\|\mathcal{S}_{\beta}\left(f_{1}\right)\right\|_{L_{w_{2}}^{q}}^{q}\right)^{p / q} \\
& \leq C \sum_{k \in \mathbb{Z}}\left[w_{1}\left(B_{k}\right)\right]^{\alpha p / n}\left\|f_{1}\right\|_{L_{w_{2}}^{q}}^{p} \\
& \leq C \sum_{k \in \mathbb{Z}}\left[w_{1}\left(B_{k}\right)\right]^{\alpha p / n}\left\|f \chi_{k}\right\|_{L_{w_{2}}^{q}}^{p} \leq C\|f\|_{\dot{K}_{q}^{\alpha, p}\left(w_{1}, w_{2}\right.}^{p} .
\end{aligned}
$$

For any $x \in C_{k}$, it follows from inequalities (33) and (34) that

$$
\begin{aligned}
\left|\mathcal{S}_{\beta}\left(f_{2}\right)(x)\right| & \leq \sum_{\ell=-\infty}^{k-2}\left|\mathcal{S}_{\beta}\left(f \chi_{\ell}\right)(x)\right| \\
& \leq C \sum_{\ell=-\infty}^{k-2} \frac{1}{|x|^{n}}\left(\int_{2^{\ell-1}<|z| \leq 2^{\ell}}|f(z)| d z\right) \\
& \leq C \sum_{\ell=-\infty}^{k-2} \frac{\left|B_{\ell}\right|}{\left|B_{k}\right|}\left[w_{2}\left(B_{\ell}\right)\right]^{-1 / q}\left\|f \chi_{\ell}\right\|_{L_{w_{2}}^{q}} .
\end{aligned}
$$


By using Lemma 9, inequality (38), and the fact that $\alpha q_{1}=$ $n\left(1-q_{2} / q\right)$, we deduce that

$$
\begin{aligned}
& \left|\mathcal{S}_{\beta}\left(f_{2}\right)(x)\right| \leq C \cdot \frac{1}{\left[w_{1}\left(B_{k}\right)\right]^{\alpha / n}\left[w_{2}\left(B_{k}\right)\right]^{1 / q}} \\
& \times \sum_{\ell=-\infty}^{k-2}\left[w_{1}\left(B_{\ell}\right)\right]^{\alpha / n}\left\|f \chi_{\ell}\right\|_{L_{w_{2}}^{q}} \cdot \frac{\left|B_{\ell}\right|}{\left|B_{k}\right|} \\
& \cdot \frac{\left[w_{2}\left(B_{k}\right)\right]^{1 / q}}{\left[w_{2}\left(B_{\ell+2}\right)\right]^{1 / q}} \cdot \frac{\left[w_{1}\left(B_{k}\right)\right]^{\alpha / n}}{\left[w_{1}\left(B_{\ell+2}\right)\right]^{\alpha / n}} \\
& \leq C \cdot \frac{1}{\left[w_{1}\left(B_{k}\right)\right]^{\alpha / n}\left[w_{2}\left(B_{k}\right)\right]^{1 / q}} \\
& \times \sum_{\ell=-\infty}^{k-2}\left[w_{1}\left(B_{\ell}\right)\right]^{\alpha / n}\left\|f \chi_{\ell}\right\|_{L_{w_{2}}^{q}} \\
& \cdot\left(\frac{\left|B_{\ell+2}\right|}{\left|B_{k}\right|}\right)^{1-\alpha q_{1} / n-q_{2} / q} \\
& \leq C \cdot \frac{1}{\left[w_{1}\left(B_{k}\right)\right]^{\alpha / n}\left[w_{2}\left(B_{k}\right)\right]^{1 / q}} \\
& \times \sum_{\ell=-\infty}^{k-2}\left[w_{1}\left(B_{\ell}\right)\right]^{\alpha / n}\left\|f \chi_{\ell}\right\|_{L_{w_{2}}^{q}} .
\end{aligned}
$$

Moreover, since $0<p \leq 1$, then we have that, for any $x \in C_{k}$,

$$
\begin{aligned}
\left|\mathcal{S}_{\beta}\left(f_{2}\right)(x)\right| \leq & C \cdot \frac{1}{\left[w_{1}\left(B_{k}\right)\right]^{\alpha / n}\left[w_{2}\left(B_{k}\right)\right]^{1 / q}} \\
& \times\left(\sum_{\ell=-\infty}^{k-2}\left[w_{1}\left(B_{\ell}\right)\right]^{\alpha p / n}\left\|f \chi_{\ell}\right\|_{L_{w_{2}}^{q}}^{p}\right)^{1 / p} \\
\leq & C \cdot \frac{1}{\left[w_{1}\left(B_{k}\right)\right]^{\alpha / n}\left[w_{2}\left(B_{k}\right)\right]^{1 / q}}\|f\|_{\dot{K}_{q}^{\alpha, p}\left(w_{1}, w_{2}\right)}
\end{aligned}
$$

Set $A_{k}=\left[w_{1}\left(B_{k}\right)\right]^{-\alpha / n}\left[w_{2}\left(B_{k}\right)\right]^{-1 / q}$. If $\left\{x \in C_{k}:\left|\mathcal{S}_{\beta}\left(f_{2}\right)(x)\right|>\right.$ $\lambda / 3\}=\emptyset$, then the inequality

$$
I_{2}^{\prime} \leq C\|f\|_{\dot{K}_{q}^{\alpha, p}\left(w_{1}, w_{2}\right)}^{p}
$$

holds trivially. Now we suppose that $\left\{x \in C_{k}:\left|\mathcal{S}_{\beta}\left(f_{2}\right)(x)\right|>\right.$ $\lambda / 3\} \neq \emptyset$. First it is easy to verify that $\lim _{k \rightarrow \infty} A_{k}=0$. Then, for any fixed $\lambda>0$, we are able to find a maximal positive integer $k_{\lambda}$ such that

$$
\frac{\lambda}{3} \leq C \cdot A_{k_{\lambda}}\|f\|_{\dot{K}_{q}^{\alpha, p}\left(w_{1}, w_{2}\right)}
$$

Hence

$$
\begin{aligned}
I_{2}^{\prime} & \leq \lambda^{p} \sum_{k=-\infty}^{k_{\lambda}}\left[w_{1}\left(B_{k}\right)\right]^{\alpha p / n}\left[w_{2}\left(B_{k}\right)\right]^{p / q} \\
& \leq C\|f\|_{\dot{K}_{q}^{\alpha, p}\left(w_{1}, w_{2}\right)}^{p} \sum_{k=-\infty}^{k_{\lambda}} \frac{\left[w_{1}\left(B_{k}\right)\right]^{\alpha p / n}}{\left[w_{1}\left(B_{k_{\lambda}}\right)\right]^{\alpha p / n}} \cdot \frac{\left[w_{2}\left(B_{k}\right)\right]^{p / q}}{\left[w_{2}\left(B_{k_{\lambda}}\right)\right]^{p / q}} .
\end{aligned}
$$

Because $B_{k} \subseteq B_{k_{\lambda}}$, then by Lemma 10, with the same notations $\delta_{i}$ as in (49), we can get

$$
\frac{w_{i}\left(B_{k}\right)}{w_{i}\left(B_{k_{\lambda}}\right)} \leq C\left(\frac{\left|B_{k}\right|}{\left|B_{k_{\lambda}}\right|}\right)^{\delta_{i}}, \quad \text { for } i=1 \text { and } 2 \text {. }
$$

Therefore

$$
\begin{aligned}
I_{2}^{\prime} & \leq C\|f\|_{\dot{K}_{q}^{\alpha, p}\left(w_{1}, w_{2}\right)}^{p} \sum_{k=-\infty}^{k_{\lambda}}\left(\frac{\left|B_{k}\right|}{\left|B_{k_{\lambda}}\right|}\right)^{\alpha \delta_{1} p / n+\delta_{2} p / q} \\
& \leq C\|f\|_{\dot{K}_{q}^{\alpha, p}\left(w_{1}, w_{2}\right)}^{p} .
\end{aligned}
$$

On the other hand, it follows from inequalities (34) and (45) that

$$
\begin{aligned}
\left|\mathcal{S}_{\beta}\left(f_{3}\right)(x)\right| & \leq \sum_{\ell=k+2}^{\infty}\left|\mathcal{S}_{\beta}\left(f \chi_{\ell}\right)(x)\right| \\
& \leq C \sum_{\ell=k+2}^{\infty} \int_{2^{\ell-1}<|z| \leq 2^{\ell}} \frac{|f(z)|}{|z|^{n}} d z \\
& \leq C \sum_{\ell=k+2}^{\infty}\left[w_{2}\left(B_{\ell}\right)\right]^{-1 / q}\left\|f \chi_{\ell}\right\|_{L_{w_{2}}^{q}} .
\end{aligned}
$$

In the present situation, since $B_{k} \subseteq B_{\ell-2}$ with $\ell \geq k+2$, then it follows from inequality (49) that

$$
\begin{aligned}
\left|\mathcal{S}_{\beta}\left(f_{3}\right)(x)\right| \leq & C \cdot \frac{1}{\left[w_{1}\left(B_{k}\right)\right]^{\alpha / n}\left[w_{2}\left(B_{k}\right)\right]^{1 / q}} \\
& \times \sum_{\ell=k+2}^{\infty}\left[w_{1}\left(B_{\ell}\right)\right]^{\alpha / n}\left\|f \chi_{\ell}\right\|_{L_{w_{2}}^{q}} \\
\leq & C \cdot \frac{\left[w_{2}\left(B_{k}\right)\right]^{1 / q}}{\left[w_{2}\left(B_{\ell-2}\right)\right]^{1 / q}} \cdot \frac{\left[w_{1}\left(B_{k}\right)\right]^{\alpha / n}}{\left[w_{1}\left(B_{\ell-2}\right)\right]^{\alpha / n}} \\
& \left.\times \sum_{\ell=k+2}^{\infty}\left[B_{k}\right)\right]^{\alpha / n}\left[w_{2}\left(B_{\ell}\right)\right]^{\alpha / n}\left\|f \chi_{\ell}\right\|_{L_{w_{2}}^{q}}^{1 / q} \\
\leq & \left.C \cdot \frac{\mid\left(B_{k} \mid\right.}{\left|B_{\ell-2}\right|}\right)^{\alpha \delta_{1} / n+\delta_{2} / q} \\
& \times \sum_{\ell=k+2}^{\infty}\left[w_{1}\left(B_{k}\right)\right]^{\alpha / n}\left[w_{2}\left(B_{k}\right)\right]^{\alpha / n}\left\|f \chi_{\ell}\right\|_{L_{w_{2}}^{q}} .
\end{aligned}
$$


Furthermore, recalling that $0<p \leq 1$, then, for any $x \in C_{k}$, we have

$$
\begin{aligned}
\left|\mathcal{\delta}_{\beta}\left(f_{3}\right)(x)\right| \leq & C \cdot \frac{1}{\left[w_{1}\left(B_{k}\right)\right]^{\alpha / n}\left[w_{2}\left(B_{k}\right)\right]^{1 / q}} \\
& \times\left(\sum_{\ell=k+2}^{\infty}\left[w_{1}\left(B_{\ell}\right)\right]^{\alpha p / n}\left\|f \chi_{\ell}\right\|_{L_{w_{2}}^{q}}^{p}\right)^{1 / p} \\
\leq & C \cdot \frac{1}{\left[w_{1}\left(B_{k}\right)\right]^{\alpha / n}\left[w_{2}\left(B_{k}\right)\right]^{1 / q}}\|f\|_{\dot{K}_{q}^{\alpha, p}\left(w_{1}, w_{2}\right)} .
\end{aligned}
$$

Repeating the arguments used for the term $I_{2}^{\prime}$, we can also obtain

$$
I_{3}^{\prime} \leq C\|f\|_{\dot{K}_{q}^{\alpha, p}\left(w_{1}, w_{2}\right)}^{p} .
$$

Combining the above estimates for $I_{1}^{\prime}, I_{2}^{\prime}$, and $I_{3}^{\prime}$ and then taking the supremum over all $\lambda>0$, we finish the proof of Theorem 4.

\section{Proofs of Theorems 5 and 6}

In order to prove the main theorems of this section, let us first establish the following results.

Proposition 11. Let $0<\beta \leq 1, q=2$, and $w \in A_{q_{2}}$ with $1 \leq q_{2} \leq q$. Then, for any $j \in \mathbb{Z}_{+}$, one has

$$
\left\|\mathcal{S}_{\beta, 2^{j}}(f)\right\|_{L_{w}^{2}} \leq C \cdot 2^{j n q_{2} / 2}\left\|\mathcal{S}_{\beta}(f)\right\|_{L_{w}^{2}} .
$$

Proof. Since $w \in A_{q_{2}}$, then, by Lemma 9, we know that for any $(y, t) \in \mathbb{R}_{+}^{n+1}$,

$$
\begin{array}{r}
w\left(B\left(y, 2^{j} t\right)\right)=w\left(2^{j} B(y, t)\right) \leq C \cdot 2^{j n q_{2}} w(B(y, t)) \\
j=1,2, \ldots
\end{array}
$$

Therefore

$$
\begin{aligned}
& \left\|\mathcal{S}_{\beta, 2^{j}}(f)\right\|_{L_{w}^{2}}^{2} \\
& \quad=\int_{\mathbb{R}^{n}}\left(\iint_{\mathbb{R}_{+}^{n+1}}\left(A_{\beta}(f)(y, t)\right)^{2} \chi_{|x-y|<2^{j} t} \frac{d y d t}{t^{n+1}}\right) w(x) d x \\
& \quad=\iint_{\mathbb{R}_{+}^{n+1}}\left(\int_{|x-y|<2^{j} t} w(x) d x\right)\left(A_{\beta}(f)(y, t)\right)^{2} \frac{d y d t}{t^{n+1}} \\
& \quad \leq C \cdot 2^{j n q_{2}} \iint_{\mathbb{R}_{+}^{n+1}}\left(\int_{|x-y|<t} w(x) d x\right)\left(A_{\beta}(f)(y, t)\right)^{2} \frac{d y d t}{t^{n+1}} \\
& \quad=C \cdot 2^{j n q_{2}}\left\|\mathcal{S}_{\beta}(f)\right\|_{L_{w}^{2}}^{2} .
\end{aligned}
$$

Taking square-roots on both sides of the above inequality, we are done.
Proposition 12. Let $0<\beta \leq 1,2<q<\infty$, and $w \in A_{q_{2}}$ with $1 \leq q_{2} \leq q$. Then, for any $j \in \mathbb{Z}_{+}$, one has

$$
\left\|\mathcal{S}_{\beta, 2^{j}}(f)\right\|_{L_{w}^{q}} \leq C \cdot 2^{j n q_{2} / 2}\left\|\mathcal{S}_{\beta}(f)\right\|_{L_{w}^{q}} \cdot
$$

Proof. For any $j \in \mathbb{Z}_{+}$and $0<\beta \leq 1$, it is easy to see that

$$
\left\|\mathcal{S}_{\beta, 2^{j}}(f)\right\|_{L_{w}^{q}}^{2}=\left\|\mathcal{S}_{\beta, 2^{j}}(f)^{2}\right\|_{L_{w}^{q / 2}} .
$$

Since $q / 2>1$, then, by duality, we have

$$
\begin{aligned}
& \left\|\mathcal{S}_{\beta, 2^{j}}(f)^{2}\right\|_{L_{w}^{q / 2}} \\
& =\sup _{\|b\|_{L_{w}(q / 2)^{\prime}} \leq 1}\left|\int_{\mathbb{R}^{n}} \mathcal{S}_{\beta, 2^{j}}(f)(x)^{2} b(x) w(x) d x\right| \\
& =\sup _{\|b\|_{L_{w}^{(q / 2)^{\prime}} \leq 1}} \mid \int_{\mathbb{R}^{n}}\left(\iint_{\mathbb{R}_{+}^{n+1}}\left(A_{\beta}(f)(y, t)\right)^{2}\right. \\
& \left.\quad \times \chi_{|x-y|<2^{j} t} \frac{d y d t}{t^{n+1}}\right) b(x) w(x) d x \mid \\
& =\sup _{\|b\|_{L_{w}^{(q / 2)^{\prime}}} \leq 1} \mid \iint_{\mathbb{R}_{+}^{n+1}}\left(\int_{|x-y|<2^{j} t} b(x) w(x) d x\right) \\
& \quad \times\left(A_{\beta}(f)(y, t)\right)^{2} \frac{d y d t}{t^{n+1}} \mid .
\end{aligned}
$$

For $w \in A_{q_{2}}$, we denote the weighted maximal operator by $M_{w}$; that is,

$$
M_{w}(f)(x)=\sup _{x \in B} \frac{1}{w(B)} \int_{B}|f(y)| w(y) d y,
$$

where the supremum is taken over all balls $B$ which contain $x$. Then, by Lemma 9 , we can get

$$
\begin{array}{rl}
\int_{|x-y|<2^{j} t} & b(x) w(x) d x \\
\leq & C \cdot 2^{j n q_{2}} w(B(y, t)) \cdot \frac{1}{w\left(B\left(y, 2^{j} t\right)\right)} \\
& \times \int_{B\left(y, 2^{j} t\right)} b(x) w(x) d x \\
\leq & C \cdot 2^{j n q_{2}} w(B(y, t)) \inf _{x \in B\left(y, 2^{j} t\right)} M_{w}(b)(x) \\
\leq & C \cdot 2^{j n q_{2}} \int_{|x-y|<t} M_{w}(b)(x) w(x) d x .
\end{array}
$$


Substituting inequality (77) into (75) and then using Hölder's inequality together with the $L_{w}^{(q / 2)^{\prime}}$ boundedness of $M_{w}$, we thus obtain

$$
\begin{aligned}
& \left\|\mathcal{S}_{\beta, 2^{j}}(f)^{2}\right\|_{L_{w}^{(q / 2)}} \\
& \quad \leq C \cdot 2^{j n q_{2}} \sup _{\|b\|_{L_{w}(q / 2)^{\prime}} \leq 1}\left|\int_{\mathbb{R}^{n}} \mathcal{S}_{\beta}(f)(x)^{2} M_{w}(b)(x) w(x) d x\right| \\
& \quad \leq C \cdot 2^{j n q_{2}}\left\|\mathcal{S}_{\beta}(f)^{2}\right\|_{L_{w}^{q / 2} \sup _{L_{w}^{(q / 2)^{\prime}}} \| 1}\left\|M_{w}(b)\right\|_{L_{w}^{(q / 2)^{\prime}}} \\
& \quad \leq C \cdot 2^{j n q_{2}}\left\|\mathcal{S}_{\beta}(f)^{2}\right\|_{L_{w}^{q / 2}} \\
& \quad=C \cdot 2^{j n q_{2}}\left\|\mathcal{S}_{\beta}(f)\right\|_{L_{w}^{q}}^{2} .
\end{aligned}
$$

This estimate together with (74) implies the desired result.

Proposition 13. Let $0<\beta \leq 1,1<q<2$, and $w \in A_{q_{2}}$ with $1 \leq q_{2} \leq q$. Then, for any $j \in \mathbb{Z}_{+}$, one has

$$
\left\|\mathcal{S}_{\beta, 2^{j}}(f)\right\|_{L_{w}^{q}} \leq C \cdot 2^{j n q_{2} / q}\left\|\mathcal{S}_{\beta}(f)\right\|_{L_{w}^{q}} \cdot
$$

Proof. We will adopt the same method given in [26]. For any $j \in \mathbb{Z}_{+}$, set $\Omega_{\lambda}=\left\{x \in \mathbb{R}^{n}: \mathcal{S}_{\beta}(f)(x)>\lambda\right\}$ and $\Omega_{\lambda, j}=\{x \in$ $\left.\mathbb{R}^{n}: \mathcal{S}_{\beta, 2^{j}}(f)(x)>\lambda\right\}$. We also set

$$
\Omega_{\lambda}^{*}=\left\{x \in \mathbb{R}^{n}: M_{w}\left(\chi_{\Omega_{\lambda}}\right)(x)>\frac{1}{2^{\left(j n q_{2}+1\right)} \cdot[w]_{A_{q_{2}}}}\right\} .
$$

Observe that $w\left(\Omega_{\lambda, j}\right) \leq w\left(\Omega_{\lambda}^{*}\right)+w\left(\Omega_{\lambda, j} \cap\left(\mathbb{R}^{n} \backslash \Omega_{\lambda}^{*}\right)\right)$. Thus, for any $j \in \mathbb{Z}_{+}$,

$$
\begin{aligned}
\left\|\mathcal{S}_{\beta, 2^{j}}(f)\right\|_{L_{w}^{q}}^{q}= & \int_{0}^{\infty} q \lambda^{q-1} w\left(\Omega_{\lambda, j}\right) d \lambda \\
\leq & \int_{0}^{\infty} q \lambda^{q-1} w\left(\Omega_{\lambda}^{*}\right) d \lambda \\
& +\int_{0}^{\infty} q \lambda^{q-1} w\left(\Omega_{\lambda, j} \cap\left(\mathbb{R}^{n} \backslash \Omega_{\lambda}^{*}\right)\right) d \lambda \\
= & \mathrm{I}+\mathrm{II} .
\end{aligned}
$$

The weighted weak type estimate of $M_{w}$ yields

$$
\mathrm{I} \leq C \cdot 2^{j n q_{2}} \int_{0}^{\infty} q \lambda^{q-1} w\left(\Omega_{\lambda}\right) d \lambda \leq C \cdot 2^{j n q_{2}}\left\|\mathcal{S}_{\beta}(f)\right\|_{L_{w}^{q}}^{q} .
$$

To estimate II, we now claim that the following inequality holds:

$$
\begin{aligned}
\int_{\mathbb{R}^{n} \backslash \Omega_{\lambda}^{*}} & \mathcal{S}_{\beta, 2^{j}}(f)(x)^{2} w(x) d x \\
\leq & C \cdot 2^{j n q_{2}} \int_{\mathbb{R}^{n} \backslash \Omega_{\lambda}} \mathcal{S}_{\beta}(f)(x)^{2} w(x) d x .
\end{aligned}
$$

Assuming the claim for the moment, then, it follows from Chebyshev's inequality and inequality (83) that

$$
\begin{aligned}
& w\left(\Omega_{\lambda, j} \cap\left(\mathbb{R}^{n} \backslash \Omega_{\lambda}^{*}\right)\right) \\
& \leq \lambda^{-2} \int_{\Omega_{\lambda, j} \cap\left(\mathbb{R}^{n} \backslash \Omega_{\lambda}^{*}\right)} \mathcal{S}_{\beta, 2^{j}}(f)(x)^{2} w(x) d x \\
& \leq \lambda^{-2} \int_{\mathbb{R}^{n} \backslash \Omega_{\lambda}^{*}} \mathcal{S}_{\beta, 2^{j}}(f)(x)^{2} w(x) d x \\
& \leq C \cdot 2^{j n q_{2}} \lambda^{-2} \int_{\mathbb{R}^{n} \backslash \Omega_{\lambda}} \mathcal{S}_{\beta}(f)(x)^{2} w(x) d x .
\end{aligned}
$$

Hence

$$
\begin{aligned}
\text { II } \leq C \cdot 2^{j n q_{2}} \int_{0}^{\infty} & q \lambda^{q-1} \\
& \times\left(\lambda^{-2} \int_{\mathbb{R}^{n} \backslash \Omega_{\lambda}} \mathcal{S}_{\beta}(f)(x)^{2} w(x) d x\right) d \lambda .
\end{aligned}
$$

Changing the order of integration yields

$$
\begin{aligned}
\mathrm{II} \leq C \cdot 2^{j n q_{2}} \int_{\mathbb{R}^{n}} \mathcal{S}_{\beta}(f)(x)^{2} \\
\quad \times\left(\int_{\left|\mathcal{S}_{\beta}(f)(x)\right|^{q}}^{\infty} \lambda^{q-3} d \lambda\right) w(x) d x \\
\leq C \cdot 2^{j n q_{2}} \frac{q}{2-q} \cdot\left\|\mathcal{S}_{\beta}(f)\right\|_{L_{w}^{q}}^{q} .
\end{aligned}
$$

Combining the above estimate (86) with (82) and taking $q$ th root on both sides, we are done. So it remains to prove inequality (83). Set $\Gamma_{2^{j}}\left(\mathbb{R}^{n} \backslash \Omega_{\lambda}^{*}\right)=\cup_{x \in \mathbb{R}^{n} \backslash \Omega_{\lambda}^{*}} \Gamma_{2^{j}}(x)$ and $\Gamma\left(\mathbb{R}^{n} \backslash\right.$ $\left.\Omega_{\lambda}\right)=\cup_{x \in \mathbb{R}^{n} \backslash \Omega_{\lambda}} \Gamma(x)$. For each given $(y, t) \in \Gamma_{2^{j}}\left(\mathbb{R}^{n} \backslash \Omega_{\lambda}^{*}\right)$, by Lemma 9, we thus have

$$
w\left(B\left(y, 2^{j} t\right) \cap\left(\mathbb{R}^{n} \backslash \Omega_{\lambda}^{*}\right)\right) \leq C \cdot 2^{j n q_{2}} w(B(y, t)) .
$$

It is not difficult to check that $w\left(B(y, t) \cap \Omega_{\lambda}\right) \leq w(B(y, t)) / 2$ and $\Gamma_{2^{j}}\left(\mathbb{R}^{n} \backslash \Omega_{\lambda}^{*}\right) \subseteq \Gamma\left(\mathbb{R}^{n} \backslash \Omega_{\lambda}\right)$. In fact, for any $(y, t) \in \Gamma_{2^{j}}\left(\mathbb{R}^{n} \backslash\right.$ $\left.\Omega_{\lambda}^{*}\right)$, there exists a point $x \in \mathbb{R}^{n} \backslash \Omega_{\lambda}^{*}$ such that $(y, t) \in \Gamma_{2^{j}}(x)$. Then we can deduce that

$$
\begin{aligned}
w\left(B(y, t) \cap \Omega_{\lambda}\right) \leq & w\left(B\left(y, 2^{j} t\right) \cap \Omega_{\lambda}\right) \\
= & \int_{B\left(y, 2^{j} t\right)} \chi_{\Omega_{\lambda}}(z) w(z) d z \\
\leq & {[w]_{A_{q_{2}}} \cdot 2^{j n q_{2}} w(B(y, t)) } \\
& \cdot \frac{1}{w\left(B\left(y, 2^{j} t\right)\right)} \int_{B\left(y, 2^{j} t\right)} \chi_{\Omega_{\lambda}}(z) w(z) d z .
\end{aligned}
$$

Note that $x \in B\left(y, 2^{j} t\right) \cap\left(\mathbb{R}^{n} \backslash \Omega_{\lambda}^{*}\right)$. So we have

$$
\begin{aligned}
w\left(B(y, t) \cap \Omega_{\lambda}\right) & \leq[w]_{A_{q_{2}}} \cdot 2^{j n q_{2}} w(B(y, t)) M_{w}\left(\chi_{\Omega_{\lambda}}\right)(x) \\
& \leq \frac{w(B(y, t))}{2} .
\end{aligned}
$$


Hence

$$
\begin{aligned}
w(B(y, t)) & =w\left(B(y, t) \cap \Omega_{\lambda}\right)+w\left(B(y, t) \cap\left(\mathbb{R}^{n} \backslash \Omega_{\lambda}\right)\right) \\
& \leq \frac{w(B(y, t))}{2}+w\left(B(y, t) \cap\left(\mathbb{R}^{n} \backslash \Omega_{\lambda}\right)\right)
\end{aligned}
$$

which is equivalent to

$$
w(B(y, t)) \leq 2 \cdot w\left(B(y, t) \cap\left(\mathbb{R}^{n} \backslash \Omega_{\lambda}\right)\right) .
$$

The above inequality implies in particular that there is a point $z \in B(y, t) \cap\left(\mathbb{R}^{n} \backslash \Omega_{\lambda}\right) \neq \emptyset$. In this case, we have $(y, t) \in \Gamma(z)$ with $z \in \mathbb{R}^{n} \backslash \Omega_{\lambda}$, which implies that $\Gamma_{2^{j}}\left(\mathbb{R}^{n} \backslash \Omega_{\lambda}^{*}\right) \subseteq \Gamma\left(\mathbb{R}^{n} \backslash \Omega_{\lambda}\right)$. Thus we obtain

$$
\begin{aligned}
& w\left(B\left(y, 2^{j} t\right) \cap\left(\mathbb{R}^{n} \backslash \Omega_{\lambda}^{*}\right)\right) \\
& \quad \leq C \cdot 2^{j n q_{2}} w\left(B(y, t) \cap\left(\mathbb{R}^{n} \backslash \Omega_{\lambda}\right)\right) .
\end{aligned}
$$

Therefore

$$
\begin{aligned}
& \int_{\mathbb{R}^{n} \backslash \Omega_{\lambda}^{*}} \mathcal{S}_{\beta, 2^{j}}(f)(x)^{2} w(x) d x \\
&= \int_{\mathbb{R}^{n} \backslash \Omega_{\lambda}^{*}}\left(\iint_{\Gamma_{2^{j}}(x)}\left(A_{\beta}(f)(y, t)\right)^{2} \frac{d y d t}{t^{n+1}}\right) w(x) d x \\
& \leq \iint_{\Gamma_{2^{j}}\left(\mathbb{R}^{n} \backslash \Omega_{\lambda}^{*}\right)}\left(\int_{B\left(y, 2^{j} t\right) \cap\left(\mathbb{R}^{n} \backslash \Omega_{\lambda}^{*}\right)} w(x) d x\right) \\
& \times\left(A_{\beta}(f)(y, t)\right)^{2} \frac{d y d t}{t^{n+1}} \\
& \leq C \cdot 2^{j n q_{2}} \iint_{\Gamma\left(\mathbb{R}^{n} \backslash \Omega_{\lambda}\right)}\left(\int_{B(y, t) \cap\left(\mathbb{R}^{n} \backslash \Omega_{\lambda}\right)} w(x) d x\right) \\
& \times\left(A_{\beta}(f)(y, t)\right)^{2} \frac{d y d t}{t^{n+1}} \\
& \leq C \cdot 2^{j n q_{2}} \int_{\mathbb{R}^{n} \backslash \Omega_{\lambda}} \mathcal{S}_{\beta}(f)(x)^{2} w(x) d x,
\end{aligned}
$$

which is exactly what we want. This completes the proof of Proposition 13.

We are now in a position to give the proofs of the main theorems.

Proof of Theorem 5. From the definition of $\mathscr{G}_{\lambda, \beta}^{*}$, we readily see that

$$
\begin{aligned}
& \left|\mathscr{G}_{\lambda, \beta}^{*}(f)(x)\right|^{2} \\
& \quad=\iint_{\mathbb{R}_{+}^{n+1}}\left(\frac{t}{t+|x-y|}\right)^{\lambda n}\left(A_{\beta}(f)(y, t)\right)^{2} \frac{d y d t}{t^{n+1}}
\end{aligned}
$$

$$
\begin{aligned}
& =\int_{0}^{\infty} \int_{|x-y|<t}\left(\frac{t}{t+|x-y|}\right)^{\lambda n}\left(A_{\beta}(f)(y, t)\right)^{2} \frac{d y d t}{t^{n+1}} \\
& +\sum_{j=1}^{\infty} \int_{0}^{\infty} \int_{2^{j-1} t \leq|x-y|<2^{j} t}\left(\frac{t}{t+|x-y|}\right)^{\lambda n} \\
& \times\left(A_{\beta}(f)(y, t)\right)^{2} \frac{d y d t}{t^{n+1}} \\
& \leq C\left[\mathcal{S}_{\beta}(f)(x)^{2}+\sum_{j=1}^{\infty} 2^{-j \lambda n} \mathcal{S}_{\beta, 2^{j}}(f)(x)^{2}\right] .
\end{aligned}
$$

Let $f \in \dot{K}_{q}^{\alpha, p}\left(w_{1}, w_{2}\right)$. We decompose $f(x)=f_{1}(x)+f_{2}(x)+$ $f_{3}(x)$ as in the proof of Theorem 3; then we have

$$
\begin{aligned}
& \left\|\mathscr{G}_{\lambda, \beta}^{*}(f)\right\|_{\dot{K}_{q}^{\alpha, p}\left(w_{1}, w_{2}\right)}^{p} \\
& \leq C \sum_{i=1}^{3} \sum_{k \in \mathbb{Z}}\left[w_{1}\left(B_{k}\right)\right]^{\alpha p / n}\left\|\mathscr{G}_{\lambda, \beta}^{*}\left(f_{i}\right) \chi_{k}\right\|_{L_{w_{2}}^{q}}^{p} \\
& \quad=J_{1}+J_{2}+J_{3} .
\end{aligned}
$$

Note that $\lambda>\max \left\{q_{2}, 3\right\} \geq \max \left\{q_{2}, 2 q_{2} / q\right\}$ when $q_{2} \leq q$. Since $w_{2} \in A_{q_{2}}$ and $1 \leq q_{2} \leq q$, then $w_{2} \in A_{q}$. Applying Propositions 11-13, Theorem A, and inequality (94), we obtain

$$
\begin{aligned}
& \left\|\mathscr{G}_{\lambda, \beta}^{*}\left(f_{1}\right)\right\|_{L_{w_{2}}^{q}} \\
& \leq C\left(\left\|\mathcal{S}_{\beta}\left(f_{1}\right)\right\|_{L_{w_{2}}^{q}}+\sum_{j=1}^{\infty} 2^{-j \lambda n / 2}\left\|\mathcal{S}_{\beta, 2^{j}}\left(f_{1}\right)\right\|_{L_{w_{2}}^{q}}\right) \\
& \quad \leq C\left\|f_{1}\right\|_{L_{w_{2}}^{q}}\left(1+\sum_{j=1}^{\infty} 2^{-j \lambda n / 2}\left[2^{j n q_{2} / 2}+2^{j n q_{2} / q}\right]\right) \\
& \leq C\left\|f_{1}\right\|_{L_{w_{2}}^{q}} .
\end{aligned}
$$

From the above estimate (96) and Lemma 9, it follows that

$$
\begin{aligned}
J_{1} & \leq C \sum_{k \in \mathbb{Z}}\left[w_{1}\left(B_{k}\right)\right]^{\alpha p / n}\left\|\mathscr{G}_{\lambda, \beta}^{*}\left(f_{1}\right)\right\|_{L_{w_{2}}^{q}}^{p} \\
& \leq C \sum_{k \in \mathbb{Z}}\left[w_{1}\left(B_{k}\right)\right]^{\alpha p / n}\left\|f_{1}\right\|_{L_{w_{2}}^{q}}^{p} \\
& \leq C \sum_{k \in \mathbb{Z}}\left[w_{1}\left(B_{k}\right)\right]^{\alpha p / n}\left\|f \chi_{k}\right\|_{L_{w_{2}}^{q}}^{p} \\
& \leq C\|f\|_{\dot{K}_{q}^{\alpha, p}\left(w_{1}, w_{2}\right)^{*}}^{p}
\end{aligned}
$$

For any $j \in \mathbb{Z}_{+}, x \in C_{k},(y, t) \in \Gamma_{2^{j}}(x)$, and $z \in\left\{2^{\ell-1}<|z| \leq\right.$ $\left.2^{\ell}\right\} \cap B(y, t)$ with $\ell \leq k-2$, then, by a simple calculation, we can easily deduce that

$$
t+2^{j} t \geq|x-y|+|y-z| \geq|x-z| \geq|x|-|z| \geq \frac{|x|}{2} .
$$


Thus, by inequality (31) and Minkowski's inequality, we get

$$
\begin{aligned}
& \left|\mathcal{S}_{\beta, 2^{j}}\left(f \chi_{\ell}\right)(x)\right| \\
& \quad=\left(\iint_{\Gamma_{2^{j}}(x)}\left(\sup _{\varphi \in \mathscr{C}_{\beta}}\left|\left(f \chi_{\ell}\right) * \varphi_{t}(y)\right|\right)^{2} \frac{d y d t}{t^{n+1}}\right)^{1 / 2} \\
& \quad \leq C\left(\int_{|x| / 2^{j+2}}^{\infty} \int_{|x-y|<2^{j} t}\left|t^{-n} \int_{2^{\ell-1}<|z| \leq 2^{\ell}}\right| f(z)|d z|^{2} \frac{d y d t}{t^{n+1}}\right)^{1 / 2} \\
& \quad \leq C\left(\int_{2^{\ell-1}<|z| \leq 2^{\ell}}|f(z)| d z\right)\left(\int_{|x| / 2^{j+2}}^{\infty} 2^{j n} \frac{d t}{t^{2 n+1}}\right)^{1 / 2} \\
& \quad \leq C \cdot 2^{3 j n / 2} \frac{1}{|x|^{n}}\left(\int_{2^{\ell-1}<|z| \leq 2^{\ell}}|f(z)| d z\right) .
\end{aligned}
$$

Moreover, by using Minkowski's inequality, (34), and (99), we obtain

$$
\begin{aligned}
& \left\|\mathcal{S}_{\beta, 2^{j}}\left(f_{2}\right) \chi_{k}\right\|_{L_{w_{2}}^{q}} \\
& \leq \sum_{\ell=-\infty}^{k-2}\left\|\mathcal{S}_{\beta, 2^{j}}\left(f \chi_{\ell}\right) \chi_{k}\right\|_{L_{w_{2}}^{q}} \\
& \leq C \cdot 2^{3 j n / 2} \sum_{\ell=-\infty}^{k-2}\left(\int_{2^{\ell-1}<|z| \leq 2^{\ell}}|f(z)| d z\right) \\
& \leq C \cdot \int_{2^{3 j n / 2}} \sum_{\ell=-\infty}^{k-2} \frac{\left|B_{\ell}\right|}{\left|B_{k}\right|} \cdot \frac{\left[w_{2}\left(B_{k}\right)\right]^{1 / q}}{\left[w_{2}\left(B_{\ell}\right)\right]^{1 / q}}\left\|f \chi_{\ell}\right\|_{L_{w_{2}}^{q}} .
\end{aligned}
$$

Consequently

$$
\begin{aligned}
J_{2} \leq C \sum_{k \in \mathbb{Z}}\left[w_{1}\left(B_{k}\right)\right]^{\alpha p / n} & \\
& \times\left(\left\|\mathcal{S}_{\beta}\left(f_{2}\right) \chi_{k}\right\|_{L_{w_{2}}^{q}}\right. \\
& \left.+\sum_{j=1}^{\infty} 2^{-j \lambda n / 2}\left\|\mathcal{S}_{\beta, 2^{j}}\left(f_{2}\right) \chi_{k}\right\|_{L_{w_{2}}^{q}}\right)^{p}
\end{aligned}
$$

where the last inequality holds under our assumption $\lambda>3$. On the other hand, for any $j \in \mathbb{Z}_{+}, x \in C_{k},(y, t) \in \Gamma_{2^{j}}(x)$, and $z \in\left\{2^{\ell-1}<|z| \leq 2^{\ell}\right\} \cap B(y, t)$ with $\ell \geq k+2$, it is easy to verify that

$$
t+2^{j} t \geq|x-y|+|y-z| \geq|x-z| \geq|z|-|x| \geq \frac{|z|}{2} .
$$

Then it follows from inequality (31) and Minkowski's inequality that

$$
\begin{aligned}
& \left|\mathcal{S}_{\beta, 2^{j}}\left(f \chi_{\ell}\right)(x)\right| \\
& \quad \leq C\left(\int_{|z| / 2^{j+2}}^{\infty} \int_{|x-y|<2^{j} t}\left|t^{-n} \int_{2^{\ell-1}<|z| \leq 2^{\ell}}\right| f(z)|d z|^{2} \frac{d y d t}{t^{n+1}}\right)^{1 / 2} \\
& \quad \leq C\left(\int_{2^{\ell-1}<|z| \leq 2^{\ell}}|f(z)| d z\right)\left(\int_{|z| / 2^{j+2}}^{\infty} 2^{j n} \frac{d t}{t^{2 n+1}}\right)^{1 / 2} \\
& \quad \leq C \cdot 2^{3 j n / 2}\left(\int_{2^{\ell-1}<|z| \leq 2^{\ell}} \frac{|f(z)|}{|z|^{n}} d z\right) .
\end{aligned}
$$

Furthermore, by Minkowski's inequality, (34), and (103), we have

$$
\begin{aligned}
\left\|\mathcal{S}_{\beta, 2^{j}}\left(f_{3}\right) \chi_{k}\right\|_{L_{w_{2}}^{q}} & \leq \sum_{\ell=k+2}^{\infty}\left\|\mathcal{S}_{\beta, 2^{j}}\left(f \chi_{\ell}\right) \chi_{k}\right\|_{L_{w_{2}}^{q}} \\
& \leq C \cdot 2^{3 j n / 2} \sum_{\ell=k+2}^{\infty} \frac{\left[w_{2}\left(B_{k}\right)\right]^{1 / q}}{\left[w_{2}\left(B_{\ell}\right)\right]^{1 / q}}\left\|f \chi_{\ell}\right\|_{L_{w_{2}}^{q}} .
\end{aligned}
$$


Therefore

$$
\begin{gathered}
J_{3} \leq C \sum_{k \in \mathbb{Z}}\left[w_{1}\left(B_{k}\right)\right]^{\alpha p / n} \\
\times\left(\left\|\mathcal{S}_{\beta}\left(f_{3}\right) \chi_{k}\right\|_{L_{w_{2}}^{q}}\right. \\
\left.+\sum_{j=1}^{\infty} 2^{-j \lambda n / 2}\left\|\mathcal{S}_{\beta, 2^{j}}\left(f_{3}\right) \chi_{k}\right\|_{L_{w_{2}}^{q}}\right)^{p} \\
\leq C \sum_{k \in \mathbb{Z}}\left[w_{1}\left(B_{k}\right)\right]^{\alpha p / n}\left(\sum_{\ell=k+2}^{\infty} \frac{\left[w_{2}\left(B_{k}\right)\right]^{1 / q}}{\left[w_{2}\left(B_{\ell}\right)\right]^{1 / q}}\left\|f \chi_{\ell}\right\|_{L_{w_{2}}^{q}}\right)^{p} \\
\times\left(1+\sum_{j=1}^{\infty} 2^{-j \lambda n / 2} \cdot 2^{3 j n / 2}\right)^{p} \\
\leq C \sum_{k \in \mathbb{Z}}\left[w_{1}\left(B_{k}\right)\right]^{\alpha p / n}\left(\sum_{\ell=k+2}^{\infty} \frac{\left[w_{2}\left(B_{k}\right)\right]^{1 / q}}{\left[w_{2}\left(B_{\ell}\right)\right]^{1 / q}}\left\|f \chi_{\ell}\right\|_{L_{w_{2}}^{q}}\right)^{p},
\end{gathered}
$$

where the last inequality also holds since $\lambda>3$. Following along the same lines as in Theorem 3, we can also show that

$$
\begin{aligned}
& J_{2} \leq C\|f\|_{\dot{K}_{q}^{\alpha, p}\left(w_{1}, w_{2}\right)}^{p}, \\
& J_{3} \leq C\|f\|_{\dot{K}_{q}^{\alpha, p}\left(w_{1}, w_{2}\right)}^{p} .
\end{aligned}
$$

Summing up the above estimates for $J_{1}, J_{2}$, and $J_{3}$, we complete the proof of Theorem 5.

Proof of Theorem 6. Let $f \in \dot{K}_{q}^{\alpha, p}\left(w_{1}, w_{2}\right)$. We set $f(x)=$ $f_{1}(x)+f_{2}(x)+f_{3}(x)$ as in the proof of Theorem 4 ; then for any given $\sigma>0$, we can write

$$
\begin{gathered}
\sigma^{p} \cdot \sum_{k \in \mathbb{Z}}\left[w_{1}\left(B_{k}\right)\right]^{\alpha p / n} w_{2}\left(\left\{x \in C_{k}:\left|\mathscr{G}_{\lambda, \beta}^{*}(f)(x)\right|>\sigma\right\}\right)^{p / q} \\
\leq \sum_{i=1}^{3} \sigma^{p} \cdot \sum_{k \in \mathbb{Z}}\left[w_{1}\left(B_{k}\right)\right]^{\alpha p / n} \\
\quad \times w_{2}\left(\left\{x \in C_{k}:\left|\mathscr{G}_{\lambda, \beta}^{*}\left(f_{i}\right)(x)\right|>\frac{\sigma}{3}\right\}\right)^{p / q} \\
=J_{1}^{\prime}+J_{2}^{\prime}+J_{3}^{\prime} .
\end{gathered}
$$

Since $\lambda>\max \left\{q_{2}, 3\right\} \geq \max \left\{q_{2}, 2 q_{2} / q\right\}$ when $q_{2} \leq q$, applying Chebyshev's inequality, Lemma 9, and (96), we obtain

$$
\begin{aligned}
J_{1}^{\prime} & \leq \sigma^{p} \cdot \sum_{k \in \mathbb{Z}}\left[w_{1}\left(B_{k}\right)\right]^{\alpha p / n}\left(\frac{3^{q}}{\sigma^{q}}\left\|\mathscr{G}_{\lambda, \beta}^{*}\left(f_{1}\right)\right\|_{L_{w_{2}}^{q}}^{q}\right)^{p / q} \\
& \leq C \sum_{k \in \mathbb{Z}}\left[w_{1}\left(B_{k}\right)\right]^{\alpha p / n}\left\|f_{1}\right\|_{L_{w_{2}}^{q}}^{p} \\
& \leq C \sum_{k \in \mathbb{Z}}\left[w_{1}\left(B_{k}\right)\right]^{\alpha p / n}\left\|f \chi_{k}\right\|_{L_{w_{2}}^{q}}^{p} \leq C\|f\|_{\dot{K}_{q}^{\alpha, p}\left(w_{1}, w_{2}\right)}^{p} .
\end{aligned}
$$

For the term $J_{2}^{\prime}$, when $x \in C_{k}$, then it follows from (94), (99), (34), and the fact $\lambda>3$ that

$$
\begin{aligned}
\left|\mathscr{G}_{\lambda, \beta}^{*}\left(f_{2}\right)(x)\right| & \\
\leq & \sum_{\ell=-\infty}^{k-2}\left|\mathscr{G}_{\lambda, \beta}^{*}\left(f \chi_{\ell}\right)(x)\right| \\
\leq & C \sum_{\ell=-\infty}^{k-2}\left(\left|\mathcal{S}_{\beta}\left(f \chi_{\ell}\right)(x)\right|+\sum_{j=1}^{\infty} 2^{-j \lambda n / 2}\left|\mathcal{S}_{\beta, 2^{j}}\left(f \chi_{\ell}\right)(x)\right|\right) \\
\leq & C\left(\sum_{\ell=-\infty}^{k-2} \frac{1}{|x|^{n}} \int_{2^{\ell-1}<|z| \leq 2^{\ell}}|f(z)| d z\right) \\
& \times\left(1+\sum_{j=1}^{\infty} 2^{-j \lambda n / 2} \cdot 2^{3 j n / 2}\right) \\
\leq & C \sum_{\ell=-\infty}^{k-2} \frac{1}{|x|^{n}}\left(\int_{2^{\ell-1}<|z| \leq 2^{\ell}}|f(z)| d z\right) \\
\leq & C \sum_{\ell=-\infty}^{k-2} \frac{\left|B_{\ell}\right|}{\left|B_{k}\right|}\left[w_{2}\left(B_{\ell}\right)\right]^{-1 / q}\left\|f \chi_{\ell}\right\|_{L_{w_{2}}^{q}} .
\end{aligned}
$$

For the last term $J_{3}^{\prime}$, when $x \in C_{k}$, by using (94), (103), (34), and the fact that $\lambda>3$, we get

$$
\begin{aligned}
& \left|\mathscr{G}_{\lambda, \beta}^{*}\left(f_{3}\right)(x)\right| \\
& \quad \leq \sum_{\ell=k+2}^{\infty}\left|\mathscr{G}_{\lambda, \beta}^{*}\left(f \chi_{\ell}\right)(x)\right| \\
& \quad \leq C \sum_{\ell=k+2}^{\infty}\left(\left|\mathcal{S}_{\beta}\left(f \chi_{\ell}\right)(x)\right|+\sum_{j=1}^{\infty} 2^{-j \lambda n / 2}\left|\mathcal{S}_{\beta, 2^{j}}\left(f \chi_{\ell}\right)(x)\right|\right) \\
& \quad \leq C\left(\sum_{\ell=k+2}^{\infty} \int_{2^{\ell-1}<|z| \leq 2^{\ell}} \frac{|f(z)|}{|z|^{n}} d z\right)\left(1+\sum_{j=1}^{\infty} 2^{-j \lambda n / 2} \cdot 2^{3 j n / 2}\right) \\
& \quad \leq C \sum_{\ell=k+2}^{\infty} \int_{2^{\ell-1}<|z| \leq 2^{\ell}} \frac{|f(z)|}{|z|^{n}} d z \\
& \quad \leq C \sum_{\ell=k+2}^{\infty}\left[w_{2}\left(B_{\ell}\right)\right]^{-1 / q}\left\|f \chi_{\ell}\right\|_{L_{w_{2}}^{q}} .
\end{aligned}
$$

The rest of the proof is exactly the same as that of Theorem 4, and we finally obtain

$$
\begin{aligned}
& J_{2}^{\prime} \leq C\|f\|_{\dot{K}_{q}^{\alpha, p}\left(w_{1}, w_{2}\right)}^{p}, \\
& J_{3}^{\prime} \leq C\|f\|_{K_{q}^{\alpha, p}\left(w_{1}, w_{2}\right)^{\alpha}}^{p} .
\end{aligned}
$$

Combining the above estimates for $J_{1}^{\prime}, J_{2}^{\prime}$, and $J_{3}^{\prime}$ and then taking the supremum over all $\sigma>0$, we conclude the proof of Theorem 6 . 


\section{Conflict of Interests}

The author declares that there is no conflict of interests regarding the publication of this paper.

\section{References}

[1] R. F. Gundy and R. L. Wheeden, "Weighted integral inequalities for nontangential maximal function, Lusin area integral, and Walsh-Paley series," Studia Mathematica, vol. 49, no. 2, pp. 107124, 1974.

[2] E. M. Stein, Singular Integrals and Differentiability Properties of Functions, Princeton University Press, Princeton, NJ, USA, 1970.

[3] B. Muckenhoupt and R. L. Wheeden, "Norm inequalities for the Littlewood-Paley function $g_{\lambda}^{*}$," Transactions of the American Mathematical Society, vol. 191, pp. 95-111, 1974.

[4] E. M. Stein, "On some functions of Littlewood-Paley and Zygmund," Bulletin of the American Mathematical Society, vol. 67, pp. 99-101, 1961.

[5] S. Y. A. Chang, J. M. Wilson, and T. H. Wolff, "Some weighted norm inequalities concerning the schrödinger operators," Commentarii Mathematici Helvetici, vol. 60, no. 1, pp. 217-246, 1985.

[6] S. Chanillo and R. L. Wheeden, "Some weighted norm inequalities for the area integral," Indiana University Mathematics Journal, vol. 36, pp. 277-294, 1987.

[7] M. Wilson, “The intrinsic square function," Revista Matematica Iberoamericana, vol. 23, no. 3, pp. 771-791, 2007.

[8] M. Wilson, Weighted Littlewood-Paley Theory and ExponentialSquare Integrability, vol. 1924 of Lecture Notes in Math, Springer, New York, NY, USA, 2007.

[9] A. K. Lerner, "Sharp weighted norm inequalities for LittlewoodPaley operators and singular integrals," Advances in Mathematics, vol. 226, no. 5, pp. 3912-3926, 2011.

[10] J. Z. Huang and Y. Liu, "Some characterizations of weighted Hardy spaces," Journal of Mathematical Analysis and Applications, vol. 363, no. 1, pp. 121-127, 2010.

[11] Y. Y. Liang, E. Nakai, D. C. Yang, and J. Q. Zhang, "Boundedness of intrinsic Littlewood-Paley functions on MusielakOrlicz Morrey and Campanato spaces," Banach Journal of Mathematical Analysis, vol. 8, pp. 221-268, 2014.

[12] Y. Y. Liang and D. C. Yang, "Intrinsic square function characterizations of Musielak-Orlicz Hardy spaces," Transactions of the American Mathematical Society. In press.

[13] H. Wang, "Intrinsic square functions on the weighted Morrey spaces," Journal of Mathematical Analysis and Applications, vol. 396, no. 1, pp. 302-314, 2012.

[14] H. Wang, "Weak type estimates for intrinsic square functions on weighted Morrey spaces," Analysis in Theory and Applications, vol. 29, pp. 104-119, 2013.

[15] H. Wang, "Boundedness of intrinsic square functions on the weighted weak Hardy spaces," Integral Equations and Operator Theory, vol. 75, no. 1, pp. 135-149, 2013.

[16] H. Wang and H. P. Liu, "The intrinsic square function characterizations of weighted Hardy spaces," Illinois Journal of Mathematics, vol. 56, no. 2, pp. 367-381, 2012.

[17] H. Wang and H. P. Liu, "Weak type estimates of intrinsic square functions on the weighted Hardy spaces," Archiv der Mathematik, vol. 97, no. 1, pp. 49-59, 2011.

[18] Y. Komori and K. Matsuoka, "Boundedness of several operators on weighted Herz spaces," Journal of Function Spaces and Applications, vol. 7, no. 1, pp. 1-12, 2009.
[19] S. Z. Lu and D. C. Yang, "The decomposition of weighted Herz space on $\mathbb{R}^{n}$ and its applications," Science in China A, vol. 38, no. 2, pp. 147-158, 1995.

[20] S. Z. Lu and D. C. Yang, "Hardy-Littlewood-Sobolev theorems of fractional integration on Herz-type spaces and its applications," Canadian Journal of Mathematics, vol. 48, no. 2, pp. 363380, 1996.

[21] S. Z. Lu, K. Yabuta, and D. C. Yang, "Boundedness of some sublinear operators in weighted Herz-type spaces," Kodai Mathematical Journal, vol. 23, no. 3, pp. 391-410, 2000.

[22] L. Tang and D. C. Yang, "Boundedness of vector-valued operators on weighted Herz spaces," Approximation Theory and its Applications, vol. 16, no. 2, pp. 58-70, 2000.

[23] B. Muckenhoupt, "Weighted norm inequalities for the Hardy maximal function," Transactions of the American Mathematical Society, vol. 165, pp. 207-226, 1972.

[24] J. Garcia-Cuerva and J. Rubio de Francia, Weighted Norm Inequalities and Related Topics, North-Holland, Amsterdam, The Netherlands, 1985.

[25] S. Z. Lu, D. C. Yang, and G. E. Hu, Herz Type Spaces and Their Applications, Science Press, Beijing, China, 2008.

[26] A. Torchinsky, Real-Variable Methods in Harmonic Analysis, Academic Press, New York, NY, USA, 1986. 


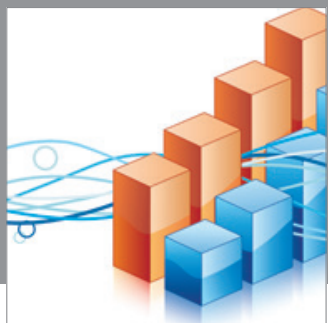

Advances in

Operations Research

mansans

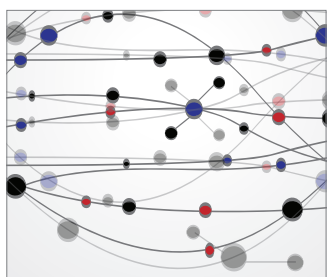

The Scientific World Journal
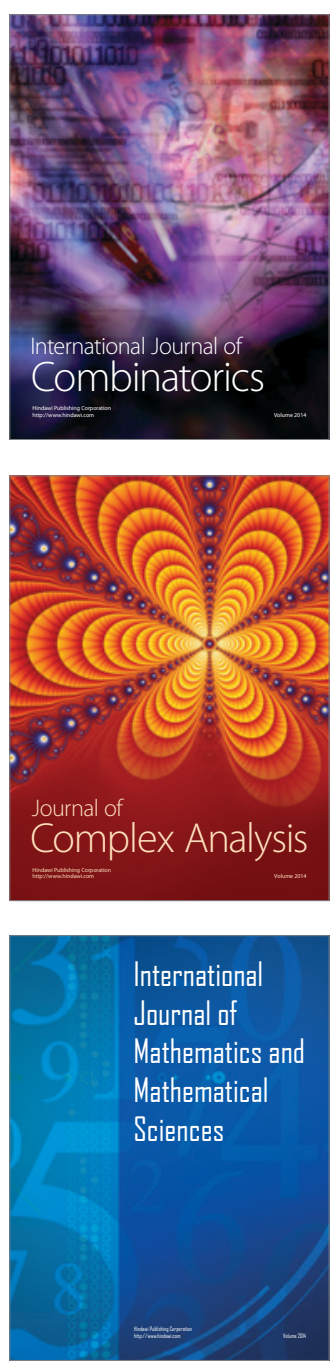
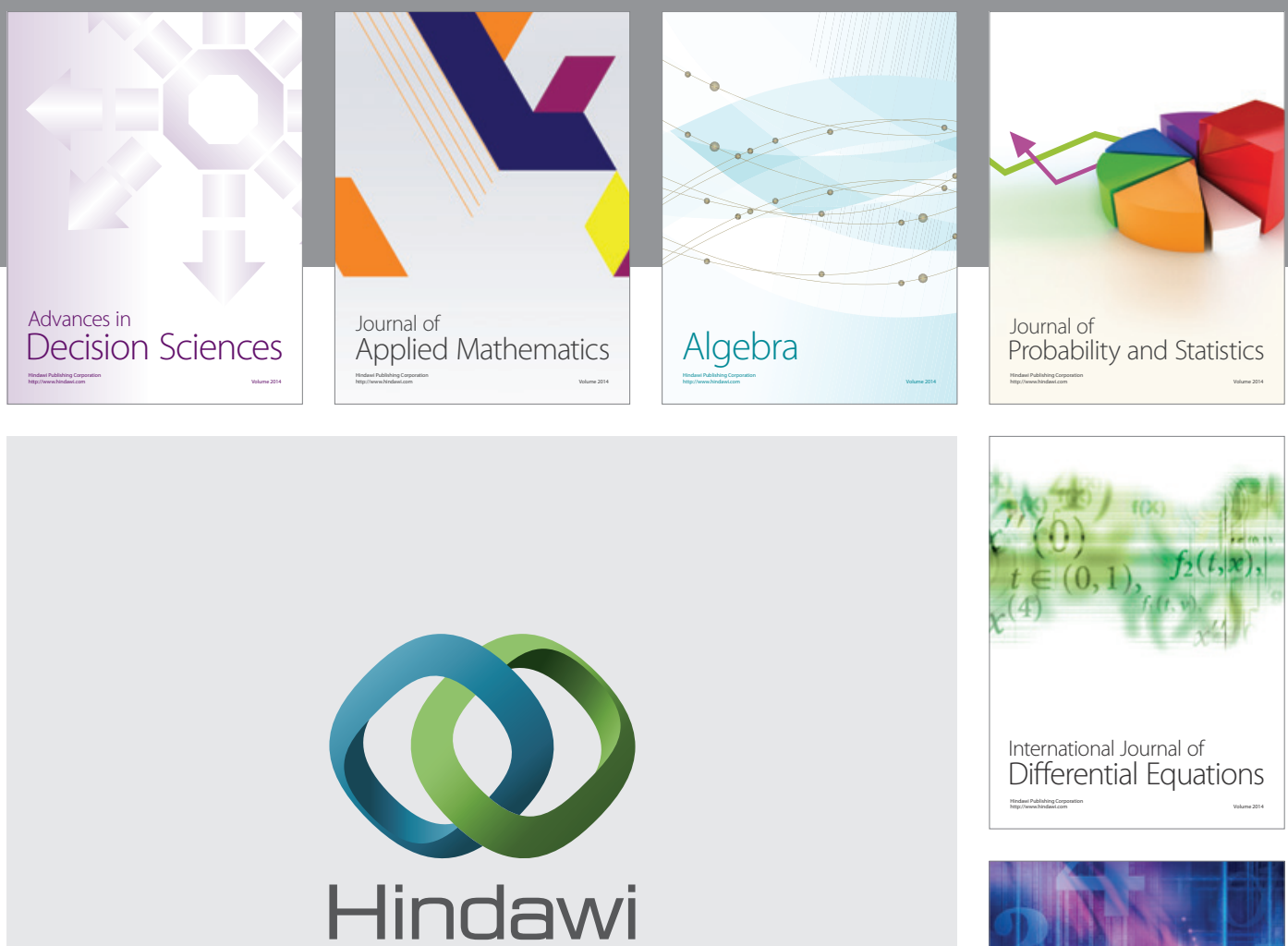

Submit your manuscripts at http://www.hindawi.com
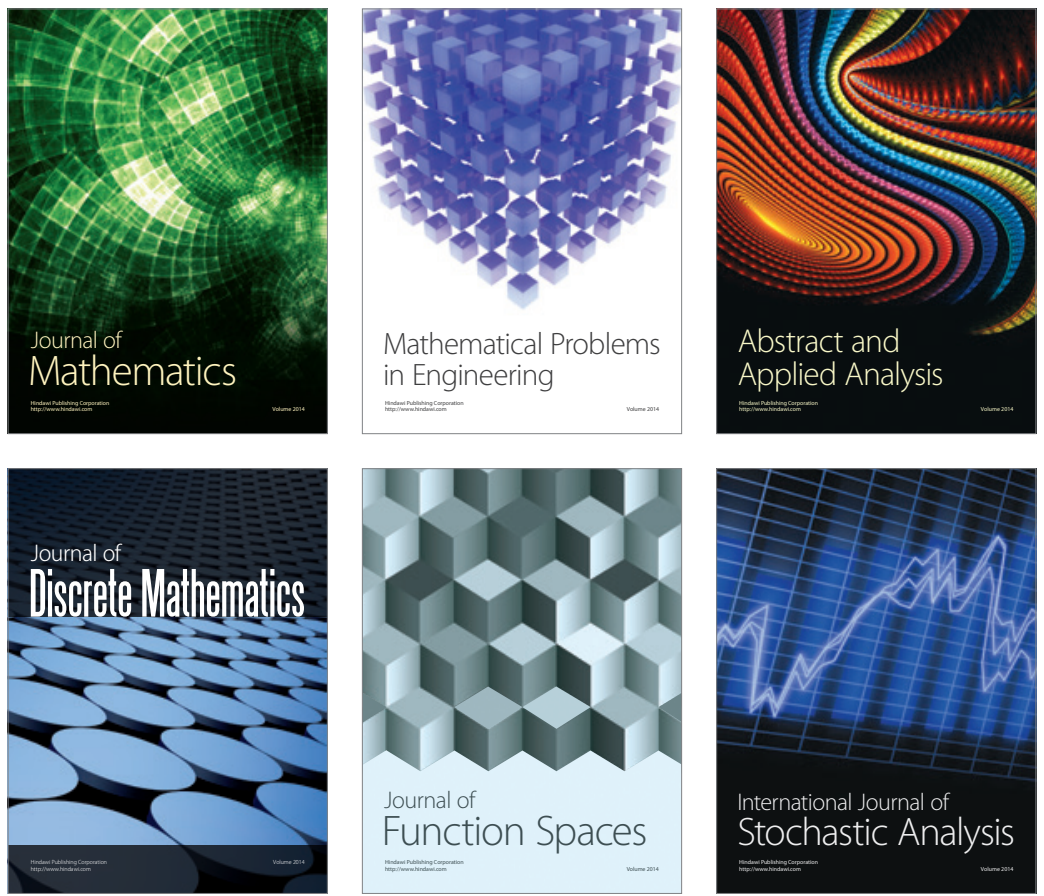

Journal of

Function Spaces

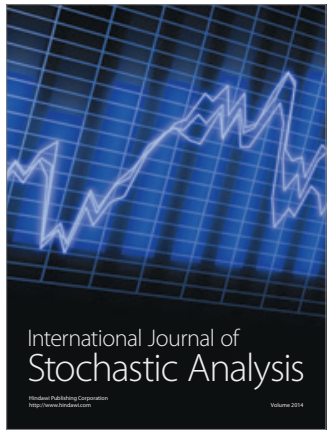

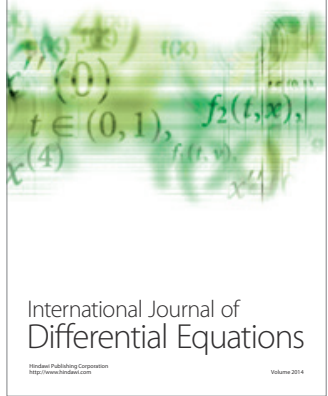
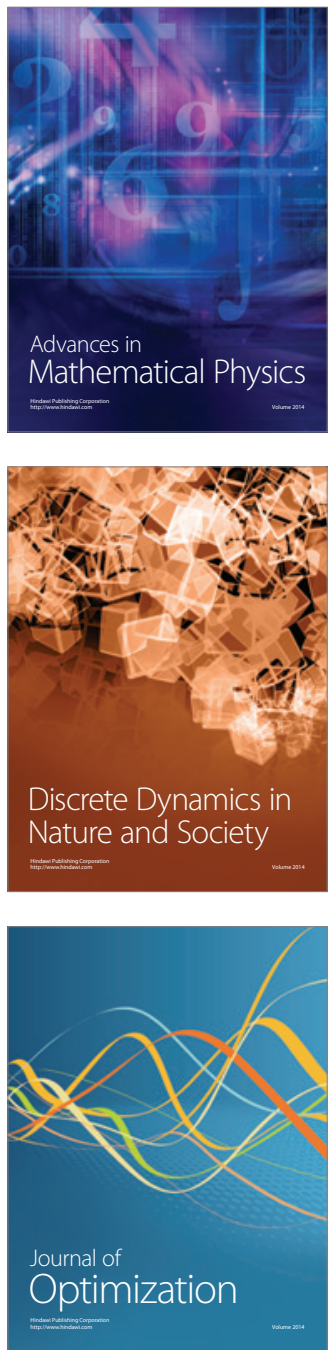